\title{
Large-scale Debloating of Binary Shared Libraries
}

\author{
IOANNIS AGADAKOS ${ }^{* \dagger}$, SRI International \\ NICHOLAS DEMARINIS ${ }^{\dagger}$, DI JIN, KENT WILLIAMS-KING, JEARSON ALFAJARDO, and \\ BENJAMIN SHTEINFELD, Brown University \\ DAVID WILLIAMS-KING, Columbia University \\ VASILEIOS P. KEMERLIS, Brown University \\ GEORGIOS PORTOKALIDIS, Stevens Institute of Technology
}

\begin{abstract}
Developers nowadays have access to an arsenal of toolkits and libraries for rapid application prototyping. However, when an application loads a library, the entirety of that library's code is mapped into the process address space, even if only a single function is actually needed. The unused portion is bloat that can negatively impact software defenses by unnecessarily inflating their overhead or increasing the attack surface. In this article, we investigate whether debloating is possible and practical at the binary level. To this end, we present Nibbler: a system that identifies and erases unused functions within dynamic shared libraries. Nibbler works in tandem with defenses such as continuous code re-randomization and controlflow integrity, enhancing them without incurring additional run-time overhead. We developed and tested a prototype of Nibbler on x86-64 Linux; Nibbler reduces the size of shared libraries and the number of available functions, for real-world binaries and the SPEC CINT2006 suite, by up to $56 \%$ and $82 \%$, respectively. We also demonstrate that Nibbler benefits defenses by showing that: (i) it improves the deployability of a continuous re-randomization system for binaries, namely, Shuffler, by increasing its efficiency by $20 \%$, and (ii) it improves certain fast but coarse and context-insensitive control-flow integrity schemes by reducing the number of gadgets reachable through indirect branch instructions by $75 \%$ and $49 \%$, on average. Last, we apply Nibbler on $\approx 30 \mathrm{~K} \mathrm{C} / \mathrm{C}++$ binaries and $\approx 5 \mathrm{~K}$ unique dynamic shared libraries (i.e., almost the complete set of the Debian sid distribution), as well as on nine official Docker images (with millions of downloads in Docker Hub), reporting entrancing findings regarding code bloat at large.
\end{abstract}

CCS Concepts: • Security and privacy $\rightarrow$ Systems security; Software and application security; Software security engineering;

Additional Key Words and Phrases: Code debloating, static binary analysis, software security

\footnotetext{
*Work done while at Stevens Institute of Technology.

${ }^{\dagger}$ Joint first authors.

This work was supported by the Office of Naval Research (ONR) and the Defense Advanced Research Projects Agency (DARPA) through awards N00014-16-1-2261, N00014-17-1-2788, and HR001118C0017. Any opinions, findings, and conclusions or recommendations expressed herein are those of the authors and do not necessarily reflect the views of the US government, ONR, or DARPA.

Authors' addresses: I. Agadakos, SRI International, 60 East 42nd Street, Suite 1520, New York, NY 10165; email: ioannis.agadakos@sri.com; N. DeMarinis, D. Jin, K. Williams-King, J. Alfajardo, B. Shteinfeld, and V. P. Kemerlis, Brown University, Box 1910, Providence, RI 02912; emails: ndemarin@cs.brown.edu, di_jin@brown.edu, vpk@cs.brown.edu; D. Williams-King, Columbia University, 500 West 120 Street, Room 450, MC0401, New York, NY 10027; email: dwk@cs.columbia.edu; G. Portokalidis, Stevens Institute of Technology, 1 Castle Point Terrace, Hoboken, NJ 07030; email: gportoka@stevens.edu.

Permission to make digital or hard copies of all or part of this work for personal or classroom use is granted without fee provided that copies are not made or distributed for profit or commercial advantage and that copies bear this notice and the full citation on the first page. Copyrights for components of this work owned by others than the author(s) must be honored. Abstracting with credit is permitted. To copy otherwise, or republish, to post on servers or to redistribute to lists, requires prior specific permission and/or a fee. Request permissions from permissions@acm.org.

(c) 2020 Copyright held by the owner/author(s). Publication rights licensed to ACM.

2576-5337/2020/12-ART19 \$15.00

https://doi.org/10.1145/3414997
}

Digital Threats: Research and Practice, Vol. 1, No. 4, Article 19. Publication date: December 2020. 
ACM Reference format:

Ioannis Agadakos, Nicholas DeMarinis, Di Jin, Kent Williams-King, Jearson Alfajardo, Benjamin Shteinfeld, David WilliamsKing, Vasileios P. Kemerlis, and Georgios Portokalidis. 2020. Large-scale Debloating of Binary Shared Libraries. Digit. Threat.: Res. Pract. 1, 4, Article 19 (December 2020), 28 pages.

https://doi.org/10.1145/3414997

\section{INTRODUCTION}

Software developers rely heavily on shared libraries for rapid application prototyping and development. However, as libraries are utilized by more and more diverse applications, they grow in complexity and size, accumulating an abundance of new features, while retaining old, potentially unused ones. When an application loads a dynamic shared library, all of this functionality is included in all of the application's processes, even if only a single function is actually used. This bloat of code affects binary programs and libraries that frequently suffer from critical vulnerabilities [24], which enable attackers to compromise them despite broadly deployed defenses, such as data execution prevention (DEP) [7] and address-space layout randomization (ASLR) [68]. More importantly, code bloat impedes the adoption of novel defenses, like continuous code re-randomization [11, 20, 97, 101] (e.g., due to increased runtime overhead), while it can also restrict the effectiveness of others, like control-flow integrity (CFI) [1] (i.e., due to over-permissiveness).

Recent work by Quach et al. [70] proposed an LLVM-based framework that analyzes code at compile time and embeds function-dependency metadata in the emitted binaries. This information is later used by a modified loader to debloat libraries, which are dynamically loaded by the application, by overwriting unused sharedlibrary code. The results of the aforementioned study confirmed that a large part of library code is indeed not needed by applications, and, therefore, it is possible to debloat them without restricting their functionality. The question this article aims to answer is the following: Is it possible to debloat binary-only software, to what extent, and what are the security benefits? Binary-only, dynamically linked or shared libraries can still be found in many settings: Commercial software is usually distributed without source code, and even open-source software may depend on (legacy) binary-only, shared libraries.

To answer this question, we design and implement Nibbler: a system that analyzes binary applications and the libraries they depend on to identify and erase unused library code. Nibbler generates thin versions of shared libraries, which can be used instead of the original, bloated ones with any of the analyzed applications. Nibbler focuses on shared libraries, as they have a series of advantages over static libraries in real-world deployments: (i) application binaries are smaller; (ii) the code of shared libraries is efficiently shared by applications, so it is not duplicated in physical memory; (iii) shared libraries can be developed in different programming languages than $\mathrm{C} / \mathrm{C}++$ (e.g., in Go) or built using different toolchains (e.g., LLVM and GCC); (iv) they facilitate maintainability (i.e., updating and patching); (v) their load-time addresses can be individually randomized by ASLR; and (vi) shared libraries with certain licenses, like (L)GPL, can be used by applications without distribution complications.

Previous attempts to debloat binaries, e.g., by Mulliner and Neugschwandtner [62], used bounded address tracking [47] to statically determine the set of used functions, which is prone to errors and requires the manual whitelisting of certain functions to avoid program crashes. In antithesis, Nibbler over-approximates the functioncall graph (FCG) of applications to conservatively include all code that could potentially be used (assuming no manual library loading). Hence, even though Nibbler also relies on static analysis, it does not lead to application crashes nor requires maintaining a whitelist.

As binary analysis is an undecidable problem, in the general case [98], we focus on non-obfuscated, compilergenerated code, and leverage symbol and relocation information-produced during compilation and/or linkingto correctly disassemble binaries. We expect that software vendors will be willing to provide (anonymized) symbols and relocations for their libraries to facilitate debloating and retrofitting defenses. More importantly, relocation information is already included in many modern libraries to support ASLR [28, 102], and various operating 
system vendors offer symbol files $[25,59,99,100]$ for their most popular libraries. If such information is not available, disassembly may still be possible using advanced reverse-engineering and binary-analysis tools [41, $64,82,92,95,96]$.

With Nibbler, we overcome various challenges pertaining to the FCG reconstruction of binaries. For example, certain compiler optimizations make transitions between functions implicit. The treatment of function pointers is another challenge, as failure to detect the usage of one could lead to incorrectly excluding used code. We propose a novel analysis for detecting address-taken (AT) functions (i.e., functions that have their address referenced as a constant) [70] that are not unused and iteratively eliminate them, while we include all others. Finally, a challenge of more technical nature is to precisely map the policies applied by the system loader when resolving symbols, which includes things like special symbols resolved based on the actual configuration of the system (e.g., the CPU model). We found that this intricacy is not addressed in earlier studies [62, 70].

We developed a prototype of Nibbler for x86-64 Linux and tested it with real-world applications, including the GNU Coreutils, the Nginx web server, the MySQL database server, and the SPEC CINT2006 benchmark suite. Our evaluation shows that Nibbler reduces library code size and functions in scope, including the notoriously hard to analyze GNU libc (glibc), by up to $56 \%$ and $82 \%$, respectively. While Nibbler does not focus on applications that manually load libraries with dlopen(), we also developed a profiling tool for collecting symbols loaded by applications at runtime, similarly to training approaches employed by earlier studies [70]. We evaluate Nibbler with runtime profiling using the Chromium browser, which extensively loads libraries at runtime. On average, we reduce code size and functions in scope by $25.98 \%$ and $34.95 \%$, respectively. In addition, we apply Nibbler on $\approx 30 \mathrm{~K} \mathrm{C} / \mathrm{C}++$ binaries and $\approx 5 \mathrm{~K}$ unique dynamic shared libraries (i.e., almost the complete set of the Debian sid distribution [86]), as well as on nine official Docker images (with millions of downloads in Docker Hub [29]), reporting entrancing findings regarding code bloat at large.

We evaluate the security benefits of debloated code by running the Nginx web server with thinned libraries under Shuffler [101], a continuous re-randomization system for binaries. We observe a throughput improvement of $20 \%$, which increases the deployability of the defense. We also developed an analysis framework to determine the effect of debloated code on certain CFI techniques [58, 90, 107, 108], including real-world CFI solutions, like Microsoft's Control-Flow Guard [58] and LLVM's CFI enforcement [90]. For coarse, context-insensitive techniques $[107,108]$, we found that the number of gadgets that can be targeted by function returns is reduced by $75 \%$ on average. The number that can be targeted by indirect function calls is reduced by $49 \%$ on average, because our analysis detects and removes unused address-taken functions. While the number of gadgets remaining in the application is still significant, the analysis performed by Nibbler clearly improves the effectiveness of some CFI defenses. Finally, we look at whether debloating can reduce attack surface by removing vulnerabilities. Unlike what is suggested by previous work [70], we argue that this type of debloating cannot reduce attack surface, as, by design, it only removes code that is never used by applications.

Below, we summarize the contributions of this article:

- We design and develop a practical system, Nibbler, which removes bloat from binary shared libraries without requiring source code and recompilation. In addition, we devise a novel method for detecting unused address-taken functions, which allows Nibbler to eliminate, safely, even more unused code.

- We evaluate the debloating capabilities of Nibbler with real-world binaries and the SPEC CINT2006 suite, and show that it removes $33 \%-56 \%$ of code from libraries and $59 \%-82 \%$ of the functions in scope. We also apply Nibbler on $\approx 30 \mathrm{~K} \mathrm{C} / \mathrm{C}++$ binaries and $\approx 5 \mathrm{~K}$ unique dynamic shared libraries, as well as on nine official Docker images, reporting interesting findings.

- We demonstrate the benefits of debloating to security schemes, like continuous code re-randomization, by integrating Nibbler with an existing system [101], observing a $20 \%$ runtime improvement for Nginx. We also demonstrate the benefits to coarse-grained CFI [58, 107, 108] by analyzing the evaluated applications and reporting that, on average, Nibbler removes $75 \%$ of the available gadgets. 
The rest of this article is organized as follows: Section 2 provides background information and motivates our work by discussing how Nibbler improves existing defenses. We present the design of Nibbler and our methodology for thinning shared libraries in Section 3. In Section 4, we briefly discuss how we implemented Nibbler and challenges we had to overcome. Section 5 presents the results from evaluating Nibbler, Section 6 discusses limitations of debloating, in general, and Section 7 summarizes related work. We conclude in Section 8.

\section{BACKGROUND AND MOTIVATION}

\subsection{Software Exploitation Techniques}

Attacks against software written in $\mathrm{C}$ and $\mathrm{C}++$ are currently employing multiple vulnerabilities to overcome defenses such as ASLR [68] and DEP [7]. They first reveal the layout of the targeted application, either by exploiting information leakage vulnerabilities [83] or using other guessing techniques [12, 38, 75, 84] to bypass ASLR. Then, they exploit memory-safety bugs to take control of code pointers, hijack control flow, and, ultimately, perform code-reuse to achieve arbitrary code execution, despite DEP [87].

Such attacks employ techniques such as ROP [80] and return-to-libc (ret2libc) [27]. The latter reuses entire functions, while the former chains arbitrary pieces of code terminating in indirect control-flow instructions, called gadgets. Other techniques, inspired by the above, include JOP [19], COP [37], COOP [78], CFB [17], and Control Jujutsu [31]. Code bloat is a boon for attackers, as more code implies potentially more gadgets to pick from, making the development of payloads easier and faster, thereby facilitating automation [18].

\subsection{Continuous Code Re-randomization}

Continuous code re-randomization techniques $[11,20,35,97,101,102]$ mitigate exploits by continuously moving code at runtime with high frequency. This introduces a real-time deadline for attackers, who only have milliseconds between exposing the layout of the process and mounting a code-reuse attack. Essentially, they aim to invalidate the leaked information before they can be used by exploits. A high re-randomization frequency can be pivotal against browser exploits [83] that utilize malicious JavaScript (JS) to execute the whole locally, using the leaked information almost immediately. Runtime overhead is also crucial, as lightweight defenses are a lot more likely to be adopted than heavyweight ones. By removing unneeded code, there is less code that needs to be shuffled at runtime, so we can improve continuous re-randomization solutions both in terms of frequency and overhead.

\subsection{Control-flow Integrity Defenses}

CFI is a technique proposed by Abadi et al. [1], which aims to enforce the control flow of the original program, forbidding arbitrary transitions. It aims to prevent the control-flow hijacking part of attacks after code pointers are taken over. There have been multiple instantiations of CFI [66, 67, 93, 107, 108] with different granularity, overhead, and requirements, but applying CFI on binaries, and achieving low overhead has been particularly problematic. The most deployable solutions enforce a coarse version of CFI $[66,107,108]$ without employing context in their enforcement of the control-flow graph (CFG). These defenses only allow functions to return to code segments that follow a function invocation (i.e., CALL-preceded gadgets) and indirect function calls to address-taken and library-exported functions (which can be called through a pointer). Nibbler enhances such CFI techniques in two ways by removing unnecessary code: (i) there are less CALL-preceded gadgets for returns to target, and (ii) there are less AT functions that can be targeted by indirect calls.

\section{DESIGN}

Nibbler is designed to primarily work with Linux ELF files [91]. We believe our techniques are applicable to other settings, such as Microsoft Windows and the PE file format [103], but leave this for future work. We focus

Digital Threats: Research and Practice, Vol. 1, No. 4, Article 19. Publication date: December 2020. 


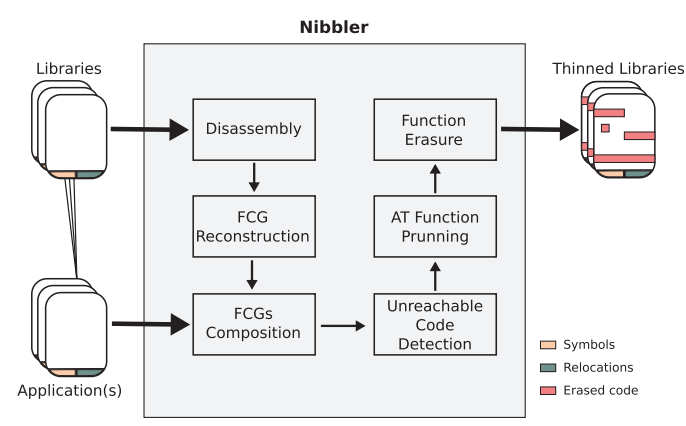

Fig. 1. Overview of Nibbler.

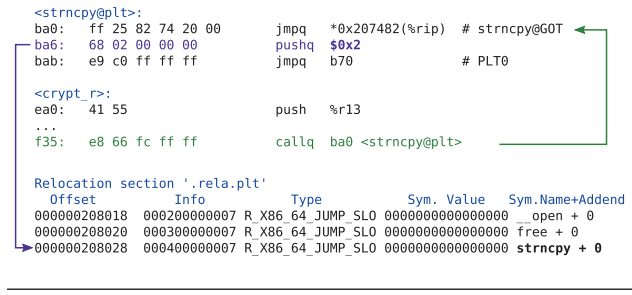

Fig. 2. Example of a call to function in a shared library (glibc) in crypt_r(), libcrypt-2.19. so.

on the x86-64 architecture, but Nibbler's requirements (disassembly, library symbols, etc.) are also available on other contemporary architectures, such as $\mathrm{x} 86, \mathrm{ARM}$, and RISC-V [102].

\subsection{Overview}

Figure 1 depicts a high-level overview of Nibbler. Given a set of binary applications, Nibbler processes the shared libraries they use, disassembles them, and statically analyzes them to reconstruct the FCG of each library. Then, the functions required by applications and the already-extracted library FCGs are composed to determine functions that are never called (i.e., unreachable code), by any of the applications of the set. At this point, Nibbler considers all functions that may be called indirectly (i.e., through a function pointer) as used. The analysis overapproximates the set of functions that could (potentially) be used to eliminate the possibility of error, assuming no manually loaded libraries (e.g., via dlopen() or dlsym()). We then perform an iterative analysis that detects AT functions that can never be used and also remove them. Finally, Nibbler produces a set of new (thinned) libraries that can be used with the input binaries, where the extra code has been erased by overwriting it with a trapping instruction [23].

\subsection{Disassembly}

Obtaining the complete disassembly of an arbitrary binary program is an undecidable problem [98]. However, modern compiler-generated binaries can be linearly disassembled (verified on GCC and LLVM [5, 8]), especially since we use symbol information to accurately identify function boundaries. Note, though, that in modern settings, we can precisely obtain the boundaries of all functions in executable ELF sections (e.g., .text, .plt), using stack unwinding information. Stack unwinding metadata are mandated by $\mathrm{C}++$ code for exception handling [55], while both GCC and LLVM emit . eh_frame sections even for C code to support interoperability with C++ [102]. Specifically, the .eh_frame section contains a Frame Description Entry (FDE) record for every function in the respective ELF [55]; the PC Begin and PC Range fields (of each FDE) provide the boundaries in question. For exported library functions, we also record the following metadata: (i) the type of the function symbol (FUNC or IFUNC); (ii) its binding, which dictates its scope (GLOBAL or WEAK, for externally visible symbols); and (iii) its version (e.g., memcpy@@GLIBC_2.14). Note that this information is always available, in shared libraries, for exported symbols.

GNU IFUNC-type symbols. These allow for the runtime selection of a target function, decided by a gateway function commonly referred to as a resolver function. Typically, this mechanism is used to select between different function implementations that use processor-specific features, such as SSE, AVX, and so on, which are more efficient but not always available. IFUNC symbols point to resolver functions, which, in turn, contain references 
to multiple targets. To avoid specializing an application to a specific environment, Nibbler preserves all possible IFUNC targets if the IFUNC symbol is called.

\subsection{Library FCG Reconstruction}

We use the disassembly and symbol information to statically reconstruct the FCG of each library. The goal is to resolve the targets of function calls. On x86, compilers use two classes of instructions to perform this task, specifically CALL and JMP instructions; we handle both in the same way. Function calls are further classified into three categories: (i) calls targeting library-local functions, (ii) calls targeting functions in other shared libraries, and (iii) indirect calls that use pointers. We ignore cases where the callee is the same with the caller (recursion). Also, multiple edges between functions are collapsed to a single one.

3.3.1 Calls to Local Functions. To resolve these calls, we go over the disassembled code and search for CALL and JMP instructions with an immediate value (i.e., a constant) as operand/argument. During execution, the CPU adds the value of the immediate to the address of the next instruction to calculate the address to transfer control to (PC-relative addressing). When the target address matches the starting address of a function, we add an edge between these two functions (caller-callee) in the FCG.

3.3.2 Calls to Functions in Shared Libraries. These calls are (usually) resolved lazily at runtime when a function is first invoked. The mechanism employed on Linux, and other Unix-like systems, uses two specially crafted sections called the Procedure Linkage Table (PLT) and the Global Offset Table (GOT) [51]. Calls to external functions are performed through the PLT. For example, in Figure 2, the call at address $0 x 0 f 35$ targets an entry in the PLT that corresponds to the strnpy() function. PLT entries are also code, which on the first invocation call the dynamic linker/loader to resolve the desired symbol. Subsequent calls direct control into the resolved function. The dynamic linker/loader (ld. so(8)) resolves external functions by name (e.g., strnpy in the example above). The name of the targeted function is indicated by the second instruction of a PLT entry-the one at address $0 \times 0$ ba 6 in Figure 2. This instruction pushes an offset in another table onto the stack, $0 \times 2$ in our example. That table essentially contains a list of references to symbol names (i.e., the string "strncpy" in our example). We have analyzed the steps taken by the loader and mirrored the steps in Nibbler to link such functions calls with symbol names. Resolution of these symbols occurs during the FCG composition step.

3.3.3 Calls Using Pointers. These function invocations are performed using a pointer, which can be dynamically computed at runtime. In binary form, they correspond to CALL or JMP instructions (with register or memory as an operand). Unfortunately, statically resolving the set of potential targets of such calls is a hard problem [52, 72]. Instead of attempting to do so and risk introducing errors, like CodeFreeze [62], Nibbler is designed to identify all the functions that could be potentially called through a pointer, and assumes that any of them may indeed be invoked. We compute the set of indirectly invoked functions by analyzing the disassembly and relocation information to identify where the address of a function is taken, and a pointer is generated. A function used as a callback, for example, will have its address taken at least once, which will add the function to the list of (all) indirect targets. This over-approximation circumvents the limitations of static analysis to accurately track pointers in memory.

In Section 3.5, we present a method for further trimming the set of indirectly invoked functions, thereby producing more tight FCGs. We employ two strategies to detect AT functions:

(1) Function pointers in disassembled code. When a program assigns a function address to a variable, instructions are generated to obtain its address and store it in memory or a register. The address of the function is either directly used as an immediate (mostly on 32-bit systems) or expressed using PC-relative addressing (x86-64). Nibbler scans the disassembled code for move (MOV) and load-effective address (LEA) instructions, looking for operands that match function addresses. Since the set of function addresses is known, this heuristic works very well, especially on x86-64 where PC-relative addressing is used extensively. However, an optimizing compiler

Digital Threats: Research and Practice, Vol. 1, No. 4, Article 19. Publication date: December 2020. 
could perform arithmetic to compute target addresses, and detecting such cases would require data-flow (e.g., value set) analysis. We do look at operands for ADD/SUB arithmetic instructions, because they may occasionally contain function references. But in our experiments, we never saw an address computation split between multiple instructions [102] (and hence data-flow analysis was not required).

(2) Function pointers via relocation information. Relocations are usually created to facilitate relocating code and data at load-time (e.g., for enabling ASLR) $[28,102]$. Each entry corresponds to a particular offset in the binary, typically a pointer to a function or global data object. Relocation entries describe how that address should be adjusted when the target entity is relocated. Modern systems support multiple relocation types [43, Chapter 4.4], which define different ways of calculating the "fix" to the targeted offset. Nibbler parses them to identify any AT functions that were not discovered in the previous step, mainly, in data sections. Relocations of type R_X86_64_IRELATIVE require more complex handling, as they specify that the targeted address should be patched based on the return value of a resolver function. Nibbler handles them by scanning the body of resolver functions, adding any code pointers identified there in the list of targets that could be potentially invoked at runtime.

\subsection{Unreachable Code Detection}

We analyze the application binaries and compose the FCGs of used libraries to conservatively estimate the library functions that are needed by the applications. Assuming the set of used libraries is known, our algorithm ensures that unused functions can be safely removed. Applications that manually load additional libraries at runtime are discussed in Section 3.7.

3.4.1 Identification of Required Symbols. At this stage, Nibbler calculates which library symbols are required by the input applications. It does so by processing them to determine the library symbols they refer to. These are obtained by scanning the PLT sections of the binaries to obtain the required symbol names, similarly to the process described in Section 3.3.2. Initialization and cleanup routines defined in libraries are also added in the set of required symbols, since they are called by the dynamic linker/loader during library loading or unloading. Such functions are defined (as arrays of function pointers) in special sections in binaries. Some of these are: .preinit_array, .init, .init_array, .fini, and .fini_array. Nibbler essentially manages to capture the non-trivial startup procedure of x86 ELF-compliant systems [42]. At this stage, we still consider that all AT functions are required.

3.4.2 Composition of Function-call Graphs. We compose the FCGs of libraries, adding edges between callers and callees, the same way the dynamic linker/loader does when a program executes. To connect the various graphs, we start by resolving each graph's calls to external functions. In the simplest case, this requires looking for a function symbol with the same name as the one referenced by a call site.

At load-time this process is performed by $1 \mathrm{~d}$. so, which enforces various rules that Nibbler replicates faithfully. In particular, we enforce the following: (a) LOCAL symbols are ignored; (b) GLOBAL symbols have precedence over WEAK ones; and (c) when the particular version of a symbol requested is not found, we use the one defined as default. Default symbols are denoted by the two " "@@' ' characters (e.g., putwchar@@GLIBC_2.2.5). (Note that there can only be one default version [30].)

When resolving symbols, it is possible that multiple symbols with the same name exist: e.g., there may be multiple local symbols with the same name, or a local symbol with the same name as a global one. For interlibrary symbol resolution, all symbols except weak or global ones are ignored. The dynamic linker/loader resolves WEAK and GLOBAL symbol references according to library load order. We did not implement all intricacies of library loading-a complex process; e.g., dependencies can be recursive-but rather create links to all weak/global functions of the same name in our graph. This approach produces a super-graph that may include more code than necessary, but is guaranteed to include all functions that could be possibly used. 


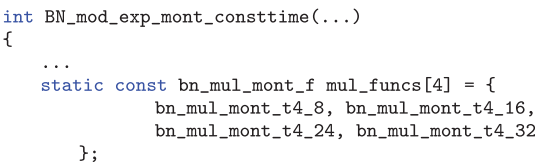

Listing 1. Example of AT functions within a function defined in crypto/bn/bn_exp.c, openssl-1.1.0j.

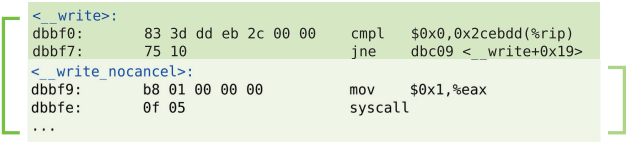

Fig. 3. Example of a fall-through function (_-write) that (re)uses the code of another function (__write_nocancel).

3.4.3 Collection of Unused Functions. At this point, we can use the composed FCG and the required symbols extracted in the previous steps to create an over-approximated set of used functions. The graph actually consists of multiple, potentially disconnected, sub-graphs; we focus on the ones that include required symbols, such as library functions invoked by one of the applications or AT functions. These nodes act as starting points that allow us to designate their whole sub-graph as used. All other functions are unreachable code that we can remove from libraries.

\subsection{AT Function Pruning}

To reduce the number of AT functions included in the FCG, we introduce an analysis that takes into account the location a code pointer was found. Initially, we separate pointers found in data (e.g., . rodata, .data) and code (i.e., . text) segments. For the latter, we iterate every function that has been classified as unused by our algorithm and check if an AT function's address is only taken within unused functions. If this condition is true, we mark the respective AT function as unused. Note that this may result in additional (function) sub-graphs to be deemed unused, and so we iteratively perform this process until no additional functions can be classified as unused.

To eliminate AT functions in data segments (e.g., in .(ro)data), we proceed as follows: First, we leverage symbol information to identify the bounds ([OBJ_BEGIN - OBJ_END]) of global data objects (i.e., symbols of type OBJECT/GLOBAL). Next, we check for relocation entries that: (a) correspond to AT functions and (b) fall within the bounds of any global object. Our approach basically identifies statically initialized arrays of function pointers or data structures that contain function pointers. Last, we iterate every function that has been classified as unused by our algorithm and check if OBJ_BEGIN is taken only within unused functions. Again, if this condition is true, we mark the AT functions that correspond to the object beginning at OBJ_BEGIN as unused, and we iteratively perform this process until no additional function sub-graphs can be classified as unused. Note that the above process is safe; it only excludes AT functions that are used by unreachable code. For example, consider the function shown in Listing 1, which defines and uses the static array of function pointers bn_mul_mont_f[]. If Nibbler detects that the function is unused, the AT functions contained in the array can also be ignored. (Assuming they are not directly invoked, nor their address is taken, by reachable code.)

\subsection{Function Erasure}

Nibbler erases functions that are not part of the application FCG by overwriting them with a single byte instruction, namely, INT3, which causes a trap and interrupts execution [23]. Both INT3 and HLT [101] are used in related work for "erasing" code; we chose INT3, as it raises a SIGTRAP, rather than a SIGSEGV, signal-SIGSEGV has many potential causes.

\subsection{Application-loaded Libraries}

Application-loaded libraries are libraries that are loaded manually through calls to dlopen(). Pointers to functions in such libraries can be dynamically retrieved using dlsym(). Although it is hard, in the general case, to statically resolve function arguments that are of pointer type [72] (mostly due to the imprecision of 
points-to analysis [72]), Nibbler can $\approx 89 \% / \approx 37 \%$ of all dlsym/dlopen arguments, across $\approx 30 \mathrm{~K} \mathrm{C} / \mathrm{C}++$ binaries and $\approx 5 \mathrm{~K}$ unique dynamic shared libraries from the Debian sid distribution [86] using standard, intra-procedural, value-tracking analysis [3, Section 9.2.5] (see Section 5.4).

Nevertheless, Nibbler cannot always safety debloat an application that calls dlsym/dlopen. In our experience, however, profiling such an application with common workloads reveals the additional dependencies, while previous studies concur with this assessment [70]. Alternatively, we can be conservative and avoid debloating applications that manually load libraries or leverage packaging semantics to include additional code in scope (e.g., all the . so files included in a particular package).

\subsection{Challenges}

Function aliases. Functions may be encompassed by multiple symbols of the same size (but often different type or scope), in effect creating aliases for the same code. We treat all aliases as a single entity and a reference to any name is sufficient to prevent the function from being removed.

Fall-through functions. Some symbols share code (or overlap) with other symbols. This requires that we employ caution when erasing an unused function, as its bytes may be shared by another symbol. A frequent case in GNU libc is fall-through functions, shown in Figure 3, where one function performs a few checks and then drops into the beginning of another function. We carefully identify each function that does not terminate in a control-flow transfer, forming a reference to the following function and preventing its removal if the previous function is used.

No-return functions. Functions that the compiler knows will terminate can be marked with the __noreturn _GCC attribute, which will be recursively propagated if possible. When generating a call to such a function, like__fortify_fail, the compiler may simply stop generating code afterwards (which would be unreachable). Luckily, we always observed the compiler generating a NOP following the CALL in this case, which allows us to avoid (incorrectly) classifying this case as a fall-through function.

GNU libc sub-libraries. While we view libc as a single library that is used by $\mathrm{C} / \mathrm{C}++$ programs, its most popular version, GNU libc (glibc), actually consists of sub-libraries that implement back-ends to common interfaces. The Name Service Switch (NSS) [53] is used by glibc to select among different name resolution mechanisms (e.g., flat-file databases, DNS, LDAP). In particular, glibc consults nsswitch. conf to determine the mapping between various databases (passwd, shadow, group, hosts, etc.) and resolution mechanisms (e.g., files, dns, ldap).

Each such mechanism corresponds to a different dynamic shared library (e.g., libnss_files.so, libnss_dns.so, libnss_ldap.so), which, in turn, provides a specific implementation of the NSS API. Depending on the contents of nsswitch.conf, glibc loads the respective . so ELF file, using dlopen, and invokes the relevant (NSS) functions indirectly after obtaining their addresses via dlsym. Nibbler parses nsswitch. conf and adds the matching ELF object(s) and function(s) in the analysis scope. Note that we also support including all sub-library symbols into Nibbler's analysis scope.

Zero-sized symbols. Certain internal functions, like _start (GCC-inserted), have a symbol of size zero. These functions have known semantics (e.g., _start calls__libc_start_main), and so we add them to the FCG for completeness, marking them as non-removable.

\section{NIBBLER IMPLEMENTATION}

We developed two separate, functionally equivalent prototypes of Nibbler on Linux. The first/older one [2] is written in Python; specifically, the disassembly and static analysis components consist of $\approx 7$ KLOC. In addition, this prototype leverages the objdump [36] Linux utility for linear disassembly and symbol information and the pyelftools [10] Python package to access ELF files. Our second/newer implementation consists of $\approx 2.5 \mathrm{KLOC}$ of $\mathrm{C} / \mathrm{C}++$ and is built atop the Egalito framework [102].

Digital Threats: Research and Practice, Vol. 1, No. 4, Article 19. Publication date: December 2020. 
Egalito is a binary recompiler, it allows rewriting binaries in-place, and it does so by first lifting binary code into a layout-agnostic, but machine-specific, intermediate representation (IR), called EIR, and then allowing "tools" to inspect or alter it, accordingly. We implemented the algorithms described in Section 3, regarding FCG reconstruction and AT function elimination, as an Egalito "pass" in the case of the newer prototype and from scratch in the case of the older one. (This is why the Python implementation is considerably larger, in terms of LOC, from the $\mathrm{C} / \mathrm{C}++$ one.) Note that we do not utilize the binary rewriting features of Egalito; we merely leverage the framework's API to precisely disassemble the corresponding binaries and lift their code in EIR form, which, in turn, we use for implementing the analyses required for constructing the FCG, identifying all AT functions, pruning unreachable parts of the call graph, and so on. We chose Egalito over similar frameworks, as it employs the best jump table analysis to date. Since by default all binaries installed on Linux are stripped of symbols, we developed a tool for fetching the debug packages of the binaries and libraries we want to process. It uses the build-id of an installed library to find a corresponding match in the debug repositories and automatically downloas it using the respective package management tools (i.e., apt (8)).

Application-loaded libraries. We implemented a tool to collect the libraries and symbols that are manually loaded by programs with dlopen(). We exploited the linker's auditing interface in Linux [54] to introduce "hooks" that are called before any operation is performed, such as searching and opening a library, resolving a symbol, etc. Specifically, we developed a shared library that sets up hooks to receive all pertinent information from the loader, filters events unrelated to dynamic loading, and logs the rest. The tool can be easily activated by setting the LD_AUDIT environment variable before running an application.

\section{EVALUATION}

To evaluate Nibbler, we used the following sets of applications:

(1) 117 unique binaries and 16 unique shared libraries, which correspond to Coreutils, Nginx, MySQL, and SPEC CINT2006, for assessing the benefits of Nibbler in terms of code reduction and security, and also compare and contrast with related studies (Section 5.1).

(2) $\approx 30 \mathrm{~K} \mathrm{C} / \mathrm{C}++$ binaries and $\approx 5 \mathrm{~K}$ unique shared libraries (i.e., almost the complete set of the Debian sid distribution [86]), which we analyze, using Nibbler, to better understand code bloat at large, reporting interesting findings pertaining to whole Linux distributions (Section 5.2).

(3) Nine official Docker images (with millions of downloads in Docker Hub), which we debloat, using Nibbler, to demonstrate the opportunities for trimming code across whole container images (Section 5.3).

Last, we discuss the performance of Nibbler, as well as its behavior with application-loaded libraries.

\subsection{Debloating Application Sets}

For an in-depth analysis of Nibbler, we use the following application suites on Debian GNU/Linux x86-64 (v9): Coreutils (v8.26), Nginx (v1.10.3), MySQL (v5.5.8), and SPEC CINT2006; we also used the stock GNU libc (v2.24). We applied Nibbler on Nginx and MySQL individually, as well as on all the binaries in Coreutils and SPEC CINT2006, ${ }^{1}$ treating them as sets of applications. In addition, we applied Nibbler on all the above applications, considering them as a single set totaling 117 binaries. The end result is five sets of thinned libraries. Note that for every application set, we generate one set of libraries to satisfy the requirements of all the included binaries.

We verified that Nibbler removes only unused code with the following tests (all completed successfully):

- Coreutils: We run the built-in high-coverage test suite, invoked through "make check."

- Nginx: We used Siege [46] to perform requests on a running server, and Nginx's official test suite.

- MySQL: We used the officially provided test suite, invoked through "mysql-test-run.pl."

- SPEC: We used the "ref" workload.

\footnotetext{
${ }^{1}$ Excluding perlbench, which did not compile successfully.

Digital Threats: Research and Practice, Vol. 1, No. 4, Article 19. Publication date: December 2020.
} 
Table 1. The Effect of Nibbler on Application Sets

\begin{tabular}{|c|c|c|c|c|c|c|c|c|}
\hline \multicolumn{3}{|c|}{ Application Set } & \multicolumn{6}{|c|}{ Code Reduction (Lib. Set) } \\
\hline \multirow{2}{*}{$\operatorname{App}(s)$} & \multicolumn{2}{|c|}{ Set Size } & \multicolumn{3}{|c|}{ Functions } & \multicolumn{3}{|c|}{ Code (KB) } \\
\hline & \# of Bin & \# of Lib. & $\mathrm{O}$ & - & $\%$ & $\mathrm{O}$ & - & $\%$ \\
\hline (a) Coreutils & 104 & 11 & 4,754 & 2,796 & $58.81 \%$ & $2,164.61$ & 711.01 & $32.85 \%$ \\
\hline (b) SPEC & 11 & 5 & 7,808 & 5,729 & $73.37 \%$ & $2,431.15$ & $1,104.93$ & $45.44 \%$ \\
\hline (c) Nginx & 1 & 7 & 8,599 & 7,015 & $81.57 \%$ & $2,917.77$ & $1,632.71$ & $55.95 \%$ \\
\hline (d) MySQL & 1 & 8 & 7,979 & 5,524 & $69.23 \%$ & $2,522.66$ & $1,010.17$ & $40.04 \%$ \\
\hline$(\mathrm{a})+(\mathrm{b})+(\mathrm{c})+(\mathrm{d})$ & 117 & 16 & 14,438 & 10,622 & $73.57 \%$ & $4,621.53$ & $2,208.93$ & $47.80 \%$ \\
\hline
\end{tabular}

The table summarizes library-code reduction in terms of code bytes and functions removed. " $\bigcirc$ " corresponds to original libraries,

"-" to removed code, and "\%" to reduction percentage.

Table 2. Code Removed from Common Libraries in Our Application Set (Coreutils, Nginx, MySQL, and SPEC CINT2006)

\begin{tabular}{l|rr|rr}
\hline \multirow{2}{*}{ Library } & \multicolumn{4}{|c}{ Unused Code } \\
\cline { 2 - 5 } & \multicolumn{2}{|c}{ Functions } & \multicolumn{2}{c}{ Bytes } \\
\hline librt & 52 & $(72.22 \%)$ & $8.88 \mathrm{~KB}$ & $(71.64 \%)$ \\
libattr & 17 & $(53.12 \%)$ & $3.35 \mathrm{~KB}$ & $(38.17 \%)$ \\
libgcc & 113 & $(66.86 \%)$ & $36.0 \mathrm{~KB}$ & $(56.10 \%)$ \\
libdl & 8 & $(33.33 \%)$ & $0.55 \mathrm{~KB}$ & $(26.70 \%)$ \\
libcrypto & 4,770 & $(93.82 \%)$ & $1,043.96 \mathrm{~KB}$ & $(83.57 \%)$ \\
libz & 83 & $(40.84 \%)$ & $40.84 \mathrm{~KB}$ & $(60.91 \%)$ \\
libpthread & 139 & $(46.80 \%)$ & $19.42 \mathrm{~KB}$ & $(36.89 \%)$ \\
libpcre & 21 & $(13.29 \%)$ & $33.26 \mathrm{~KB}$ & $(10.09 \%)$ \\
libc & 1,539 & $(53.27 \%)$ & $297.90 \mathrm{~KB}$ & $(24.93 \%)$ \\
libm & 426 & $(66.56 \%)$ & $172.16 \mathrm{~KB}$ & $(39.76 \%)$ \\
libstdc++ & 2,719 & $(71.31 \%)$ & $309.14 \mathrm{~KB}$ & $(45.04 \%)$ \\
libgmp & 438 & $(66.46 \%)$ & $176.60 \mathrm{~KB}$ & $(46.43 \%)$ \\
libselinux & 225 & $(63.20 \%)$ & $50.51 \mathrm{~KB}$ & $(53.13 \%)$ \\
libacl & 42 & $(60.00 \%)$ & $11.75 \mathrm{~KB}$ & $(69.99 \%)$ \\
libcap & 21 & $(75.00 \%)$ & $3.38 \mathrm{~KB}$ & $(49.97 \%)$ \\
libcrypt & 9 & $(23.08 \%)$ & $0.91 \mathrm{~KB}$ & $(4.29 \%)$ \\
\hline
\end{tabular}

5.1.1 Code Reduction. Table 1 summarizes the code reduction achieved by Nibbler on the application sets that do not manually load libraries. It performs the best with Nginx, where $55.95 \%$ of library code, in terms of bytes, is removed. However, for Coreutils, which includes a large number of diverse utilities, we are able to eliminate $32.85 \%$ of library code. If we combine all 117 applications (last row), we achieve a reduction of $47.80 \%$. If we instead focus on removed functions, reduction is between $58.81 \%-81.57 \%$, as many smaller functions are removed.

Bloat is not equally distributed in libraries. In the worst case (libcrypto), we found that $93.82 \%$ of its functions are not used by any of the four application sets. In the best case (libpcre), we removed $13.29 \%$ of its functions, however, this was also one of the smallest libraries in our set. Table 2 highlights the per-library code reduction achieved by Nibbler in libraries used by all four application sets. We think that these results demonstrate the heavy bloat in certain libraries, making their thinned versions great candidates even for system-wide replacement. 


\subsubsection{Comparison with Piece-wise.}

Debloating. While direct comparison with Piece-wise is not possible, because Quach et al. focus on debloating individual programs, we highlight our differences using Coreutils and SPEC to provide some perspective to the reader. The mean reduction, in the number of functions, that Piece-wise achieves, with respect to these two program sets, is $79 \%$ and $85 \%$, respectively; Nibbler achieves $58.81 \%$ and $73.37 \%$. This difference is primarily due to the fact that we debloat libraries for sets of applications, instead of individual binaries. In addition, the lack of semantics in binary code prevents us from effectively using analyses to eliminate more AT functions, while we preserve multiple versions of a used symbol in thinned libraries (Piece-wise keeps only one); in Section 5.2, we revisit this issue.

Memory overhead. Nibbler can be applied on an entire system or on smaller sets of applications. In the latter case, if the original version of a library is also in use, then two versions of the same library will be present in memory (i.e., vanilla and thinned library). Calculating exactly how much memory overhead the thinned library will impose is not straightforward, as the OS dynamically pages-in code pages used by applications. We can calculate, however, the additional memory required when all library code is used (worst-case analysis). This corresponds to all code pages (of the thinned library) that have at least one byte of code that was not erased.

However, Piece-wise keeps a single version of each library on disk and removes code at load time. As a result, each memory page that has code erased-but not removed entirely-is no longer shared with any other executing application that uses the same library, due to copy-on-write (COW). Consequently, the overhead increases as more applications execute concurrently. This includes multiple invocations of the same application, but not multiple processes resulting from a fork() system call. As a reference, there are approximately 39 distinct applications running in a fresh installation of Debian v9. Comparison of memory overheads is shown in Table 3. We estimate the per-invocation overhead of Piece-wise using the code-reduction numbers of Nibbler (more code removed likely means higher overhead for Piece-wise). Our approach incurs lower and more predictable memory overhead.

Load time. Nibbler does not incur any load-time overhead. In contrast, Piece-wise, which only removes code at load time, reports a $20 x$ slowdown on average with the small programs in Coreutils (20 ms on average, for a process usually requiring less than $2 \mathrm{~ms}$ ).

\subsubsection{Security Analysis.}

Impact on continuous code re-randomization. By identifying and removing unused code, Nibbler can reduce the overhead of certain security techniques, thereby easing their adoption and improving software security. Shuffler [101] is a system realizing such a technique: continuous re-randomization of a target program and all its libraries, including its own code. It does so asynchronously, from a background thread, preparing a new copy of the code every $20 \mathrm{~ms}$ (in case of Nginx), and then signaling all other threads to migrate to this new copy. Because of this asynchronous design, all functions must be re-randomized during each shuffle period, as the system cannot determine in advance what will be required. Nibbler's library thinning can combine excellently with Shuffler's defense.

We fused Nibbler with Shuffler, on Nginx v1.4.6, trimming functions that would never be used during execution, reducing the amount of work that Shuffler must perform. Overall, we nibbled $\approx 1.6 \mathrm{~K}$ functions (out of $\approx 6.2 \mathrm{~K}$ ) or $26 \%$. In our experiment, we used four Nginx worker processes pinned to two CPU cores; Shuffler threads (one per worker) were also pinned to the same cores. Shuffler's asynchronous overhead will take CPU time away from the target program, reflecting in a throughput drop. We ran 32 client threads (using the benchmark tool Siege [46]) pinned to four other cores on the same system, which was sufficient to saturate the server. This experiment is a smaller-scale version of the Nginx experiment included in the original paper [101]. Results are shown in Figure 4.

Nibbler+Shuffler performance improves when there are more Shuffler workers, and hence more CPU time is being spent on asynchronously copying code. In the 2-cores, 1-worker case, one core runs the Nginx worker and

Digital Threats: Research and Practice, Vol. 1, No. 4, Article 19. Publication date: December 2020. 
Table 3. Memory Overhead (KB) Comparison

\begin{tabular}{l|c|c}
\hline Application Set & $\begin{array}{c}\text { Nibbler } \\
\text { Max. Total }\end{array}$ & $\begin{array}{c}\text { Piece-wise [70] } \\
\text { Estimate } \\
\text { per execution }\end{array}$ \\
\hline (a) Coreutils & $1,900 \mathrm{~KB}$ & $1,024 \mathrm{~KB}$ \\
(b) SPEC & $1,148 \mathrm{~KB}$ & $580 \mathrm{~KB}$ \\
(c) Nginx & $1,668 \mathrm{~KB}$ & $1,292 \mathrm{~KB}$ \\
(d) MySQL & $2,108 \mathrm{~KB}$ & $1,248 \mathrm{~KB}$ \\
(a) + (b) +(c) + (d) & $3,256 \mathrm{~KB}$ & $1,816 \mathrm{~KB}$ \\
\hline
\end{tabular}

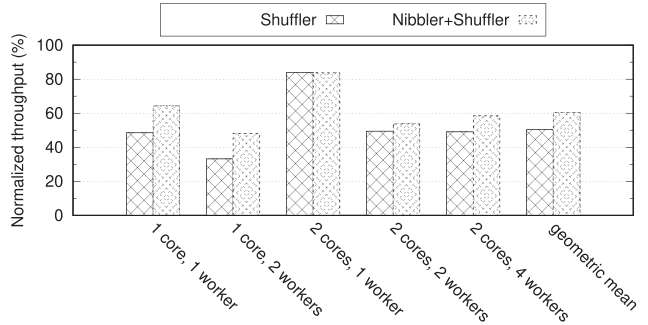

Fig. 4. Nginx throughput for vanilla Shuffler, and Nibbler+Shuffler, over an undefended baseline.

one executes the Shuffler thread, so Nibbler has little impact. However, in a carefully provisioned system with few spare resources, Nibbler improves Shuffler's performance significantly.

Specifically, the geometric mean of throughput improved from $50.51 \%$ (Shuffler) to $60.54 \%$, a relative increase of $19.87 \%$. This makes sense, since we trimmed $26 \%$ of the code; due to Shuffler's design, we expect a linear increase in performance as the amount of code decreases. Additionally, we expect these results to scale to larger experiments, since Shuffler spends very little time on coordination (0.3\% of overall runtime [101]). If the server is multiprocess, every process gets its own independent Shuffler thread, and Nibbler still reduces the overhead linearly. If the server is multithreaded, Shuffler's overhead decreases as more cores become available, and Nibbler still reduces the overhead proportionally.

Impact on control-flow integrity. Nibbler improves low-overhead, coarse CFI schemes in two ways. First, it reduces the number of (CALL-preceded) ROP gadgets that are accessible to attackers. Second, it reduces the number of functions that can be targeted by indirect functions calls by eliminating function pointers that are never used in applications (Section 3.5). To quantify this gain, we built a gadget analysis framework atop the Capstone disassembler [71] to calculate the reduction of CFI-resistant [104] gadgets in thinned libraries. Given an x86-64 ELF binary as input, our tool identifies its executable sections by parsing the respective ELF header(s), and proceeds as follows:

First, it pinpoints all the byte sequences (in the previously identified executable sections) that correspond to RET, indirect JMP, and CALL instructions; the location of every such instruction is marked as GAD_END, because it indicates the end of a gadget, while the instruction opcode (RET, JMP, CALL) specifies the type of the gadget (i.e., ROP, JOP, or COP). Second, GAD_BEGIN is set to GAD_END - 1, and Capstone is used to linearly disassemble the region [GAD_BEGIN, GAD_END]; every resulting code snippet is by definition a gadget (as it ends with an indirect branch instruction), and the type of the instruction that starts at GAD_BEGIN is used to further classify the whole gadget (more about this below). Next, GAD_BEGIN is set to GAD_END - 2, and step 2 is repeated; the process is executed recursively for GAD_BEGIN = GAD_END - 3, ., GAD_BEGIN = GAD_END - $\mathrm{k}$, where $\mathrm{k}$ (bytes) is an input parameter, typically set to 10 , in accordance to modern automated gadget-finding tools, like ROPgadget [76], Ropper [77], xrop [16], and rop-tool [88]. (We had to develop our own analysis framework, as none of the aforementioned gadget-finding tools is intended for quantitative analyses [104].)

The above procedure discovers instruction (sub)sequences, of size $1,2, \ldots, \mathrm{k}$ bytes, which are prefixes of an indirect branch, thereby constituting gadgets. For example, Imov (\%rdi), \%rax; pop $r 14$; pop r15; pop rbp; ret | will be accounted as 4 separate ROP gadgets: (a) |pop rbp; ret|, (b) |pop r15; pop rbp; ret|, (c) |pop r14; pop r15; pop rbp; ret|, and (d) |mov (\%rdi), \%rax ... ret|. Also, gadgets (a)-(c) will be classified as loading a register with a value from the stack, whereas (d) will be classified as a memory load. Last, gadgets that include invalid instruction sequences, such as privileged instructions ( $\mathrm{hlt}$, in/out, rdmsr/wrmsr, etc.), instructions that access non-general-purpose, registers (dr\#, cr\#), and vendor-specific ISA extensions (MMX, SSE, AVX, TSX), are all filtered out. 
Table 4. CFI-resistant Gadget Reduction Results

\begin{tabular}{|c|c|c|c|c|c|c|c|c|c|c|c|c|c|c|c|c|}
\hline & \multirow{3}{*}{ Suite } & \multirow{3}{*}{ Total } & \multicolumn{14}{|c|}{ Gadget Type } \\
\hline & & & \multicolumn{2}{|c|}{ Stack } & \multicolumn{2}{|c|}{ Load Reg. } & \multicolumn{2}{|c|}{ Memory } & \multicolumn{2}{|c|}{ Arithmetic } & \multicolumn{2}{|c|}{ Logic } & \multicolumn{2}{|c|}{ Branch } & \multirow[t]{2}{*}{ Syscall } & \multirow[t]{2}{*}{ NOP } \\
\hline & & & Pivot & Lift & Reg. & Stack & Load & Store & Reg. & Mem. & Reg. & Mem. & jmp & call & & \\
\hline \multicolumn{17}{|c|}{ SPEC } \\
\hline libpthread & $(92.79 \%)$ & $.0 \%$ & $\mathrm{~N} / \mathrm{A}$ & $\mathrm{N} / \mathrm{A}$ & $95.7 \%$ & $\underline{100.0 \%}$ & $\underline{100.0 \%}$ & $86.5 \%$ & $97.5 \%$ & $\underline{100.0 \%}$ & $\underline{100.0 \%}$ & $\underline{100.0 \%}$ & $\mathrm{~N} / \mathrm{A}$ & $\mathrm{N} / \mathrm{A}$ & $88.4 \%$ & $97.5 \%$ \\
\hline libm & $(39.76 \%)$ & $3 \%$ & $\mathrm{~N} / \mathrm{A}$ & N/A & N/A & $18.5 \%$ & $0.0 \%$ & $0.0 \%$ & $50.7 \%$ & $0.0 \%$ & $\overline{100.0 \%}$ & $\mathrm{~N} / \mathrm{A}$ & $\mathrm{N} / \mathrm{A}$ & N/A & N/A & $57.7 \%$ \\
\hline libc & $(42.01 \%)$ & $.8 \%$ & $91.7 \%$ & $0.0 \%$ & $75.6 \%$ & $78.0 \%$ & $72.6 \%$ & $66.2 \%$ & $75.9 \%$ & $74.7 \%$ & $62.6 \%$ & $76.9 \%$ & $25.0 \%$ & $62.1 \%$ & $3.3 \%$ & $79.7 \%$ \\
\hline bgcc & $(72.35 \%)$ & $.7 \%$ & $0.0 \%$ & N/A & $39.8 \%$ & $60.3 \%$ & $21.1 \%$ & $11.1 \%$ & $69.8 \%$ & N/A & $40.0 \%$ & N/A & N/A & N/A & J/A & $59.4 \%$ \\
\hline$c++$ & $(48.88 \%)$ & $80.1 \%$ & $0.0 \%$ & $50.0 \%$ & $48.3 \%$ & $79.5 \%$ & $76.4 \%$ & $69.7 \%$ & $72.1 \%$ & $77.8 \%$ & $44.8 \%$ & $100.0 \%$ & $100.0 \%$ & $35.7 \%$ & N/A & $78.6 \%$ \\
\hline \multicolumn{17}{|c|}{ Coreutils } \\
\hline ibpth & $94 \%)$ & $56.8 \%$ & $\mathrm{~N} / \mathrm{A}$ & $\mathrm{N} / \mathrm{A}$ & $61.1 \%$ & $57.7 \%$ & $62.5 \%$ & $56.8 \%$ & $42.5 \%$ & $\underline{100.0 \%}$ & $55.8 \%$ & $.0 \%$ & $\mathrm{~N} / \mathrm{A}$ & $\mathrm{N} / \mathrm{A}$ & $55.8 \%$ & $56.2 \%$ \\
\hline dl & $41 \%)$ & $.8 \%$ & $\mathrm{~N} / \mathrm{A}$ & $\mathrm{N} / \mathrm{A}$ & $\underline{100.0 \%}$ & $\underline{100.0 \%}$ & $\underline{100.0 \%}$ & $\underline{100.0 \%}$ & $93.8 \%$ & $\mathrm{~N} / \mathrm{A}$ & $\underline{100.0 \%}$ & A & $\mathrm{N} / \mathrm{A}$ & $100.0 \%$ & $\mathrm{~J} / \mathrm{A}$ & 96. \\
\hline inux & $(53.13 \%)$ & $.3 \%$ & $\mathrm{~N} / \mathrm{A}$ & $\mathrm{N} / \mathrm{A}$ & $80.7 \%$ & $64.0 \%$ & $90.0 \%$ & $66.7 \%$ & $76.1 \%$ & $\mathrm{~N} / \mathrm{A}$ & $78.4 \%$ & /A & N/A & $94.4 \%$ & N/A & $67.2 \%$ \\
\hline libacl & $(69.99 \%)$ & $58.8 \%$ & N/A & N/A & $63.9 \%$ & $71.7 \%$ & N/A & $60.0 \%$ & $36.8 \%$ & $100.0 \%$ & $57.9 \%$ & J/A & N/A & N/A & N/A & $61.1 \%$ \\
\hline li & $(71.64 \%)$ & $.4 \%$ & N/A & N/A & $64.8 \%$ & $76.3 \%$ & $60.0 \%$ & $50.0 \%$ & $65.4 \%$ & $\mathrm{~N} / \mathrm{A}$ & $62.5 \%$ & $0 \%$ & N/A & $66.7 \%$ & $7 \%$ & $78.1 \%$ \\
\hline pcre & $(10.15 \%)$ & $.1 \%$ & N/A & N/A & $25.0 \%$ & $16.9 \%$ & $10.0 \%$ & $30.0 \%$ & $7.7 \%$ & N/A & $33.3 \%$ & N/A & $50.0 \%$ & $0.0 \%$ & J/A & $21.4 \%$ \\
\hline c & 8\%) & $.8 \%$ & $50.0 \%$ & $0.0 \%$ & $63.5 \%$ & $56.7 \%$ & $58.8 \%$ & $55.5 \%$ & $62.6 \%$ & $63.7 \%$ & $44.6 \%$ & $5 \%$ & $25.0 \%$ & $57.6 \%$ & $7 \%$ & $65.3 \%$ \\
\hline & $\%)$ & $.4 \%$ & N/A & N/A & $50.0 \%$ & $100.0 \%$ & $100.0 \%$ & $100.0 \%$ & $45.5 \%$ & $100.0 \%$ & $100.0 \%$ & A & N/A & /A & A & $38.5 \%$ \\
\hline & $(46$ & $.5 \%$ & N/A & N/A & $6.3 \%$ & $59.6 \%$ & $79.7 \%$ & $73.8 \%$ & $71.4 \%$ & $38.9 \%$ & & $\%$ & 100 & & /A & $70.9 \%$ \\
\hline & $(9$ & $.0 \%$ & $80.0 \%$ & N/A & $5 \%$ & 100 & $\%$ & & $96.2 \%$ & A & & & $\mathrm{N}$ & & A & 96. \\
\hline p & $(49$. & $60.2 \%$ & N/A & $\mathrm{N} / \mathrm{A}$ & $40.9 \%$ & $0 \%$ & /A & $87.5 \%$ & $61.5 \%$ & $\mathrm{~N} / \mathrm{A}$ & $40.0 \%$ & 721 & $\mathrm{~N} / \mathrm{A}$ & $N / \Lambda$ & N/A & $61.9 \%$ \\
\hline \multicolumn{17}{|c|}{ MySQL } \\
\hline libm & 5\%) & $52.8 \%$ & N/A & N/A & /A & $\%$ & $\%$ & $0.0 \%$ & $52.2 \%$ & $0 \%$ & $100.0 \%$ & & $\mathrm{~N}$ & I/A & J/A & $58.9 \%$ \\
\hline thread & $(34.33 \%)$ & $.4 \%$ & N/A & N/A & $53.5 \%$ & $54.2 \%$ & $62.5 \%$ & $43.2 \%$ & $37.5 \%$ & $100.0 \%$ & $51.2 \%$ & $0.0 \%$ & $\mathrm{~N}$ & $\mathrm{~J} / \mathrm{A}$ & $5 \%$ & 46. \\
\hline libz & $(29.37 \%)$ & $.5 \%$ & N/A & N/A & $53.1 \%$ & $62.0 \%$ & $37.0 \%$ & $60.0 \%$ & $52.9 \%$ & $50.0 \%$ & $72.7 \%$ & A & N/A & $37.5 \%$ & N/A & $56.2 \%$ \\
\hline l1 & $3 \%)$ & $.0 \%$ & $91.7 \%$ & $0.0 \%$ & $67.0 \%$ & $66.4 \%$ & $64.3 \%$ & $56.3 \%$ & $67.6 \%$ & $63.2 \%$ & $54.7 \%$ & 61. & $25.0 \%$ & $50.0 \%$ & $\%$ & $72.5 \%$ \\
\hline & \%) & $42.3 \%$ & $0.0 \%$ & $\mathrm{~N} / \mathrm{A}$ & $39.8 \%$ & $\%$ & 1 & $1.1 \%$ & $66.0 \%$ & A & $\%$ & A & N/A & A & A & $56.2 \%$ \\
\hline & $(4$ & 77.5\% & $0.0 \%$ & $0.0 \%$ & $.2 \%$ & & 7 & $\%$ & $69.1 \%$ & $\%$ & $37.9 \%$ & $100.0 \%$ & $100.0 \%$ & & A & 76. \\
\hline & & $40.2 \%$ & J/A & & & & & & & & & & & & 1 & $41.9 \%$ \\
\hline libcrypt & $(4.30 \%)$ & $20.2 \%$ & N/A & N/A & $25.0 \%$ & $20.7 \%$ & $\mathrm{~N} / \mathrm{A}$ & $0.0 \%$ & $21.4 \%$ & N/A & N/A & N/A & N/A & N/A & N/A & $23.1 \%$ \\
\hline \multicolumn{17}{|c|}{ Ngin $x$} \\
\hline libpthread & $(47.75 \%)$ & $50.3 \%$ & N/A & N/A & $1.2 \%$ & $47.9 \%$ & $62.5 \%$ & $54.1 \%$ & $43.8 \%$ & $\underline{100.0 \%}$ & $41.9 \%$ & $0 \%$ & N/A & N/A & $46.5 \%$ & $55.0 \%$ \\
\hline libz & $(60.91 \%)$ & $77.2 \%$ & N/A & N/A & $68.8 \%$ & $87.0 \%$ & $51.9 \%$ & $64.0 \%$ & $67.6 \%$ & $50.0 \%$ & $81.8 \%$ & N/A & N/A & $50.0 \%$ & N/A & $78.1 \%$ \\
\hline libc & $(40.78 \%)$ & $73.8 \%$ & $91.7 \%$ & $0.0 \%$ & $71.0 \%$ & $74.0 \%$ & $71.8 \%$ & $70.2 \%$ & $74.8 \%$ & $70.9 \%$ & $62.6 \%$ & $69.2 \%$ & $25.0 \%$ & $62.1 \%$ & $60.0 \%$ & $79.2 \%$ \\
\hline libdl & $(26.70 \%)$ & $40.2 \%$ & N/A & N/A & $42.9 \%$ & $31.8 \%$ & $0.0 \%$ & $33.3 \%$ & $50.0 \%$ & N/A & $0.0 \%$ & N/A & N/A & $50.0 \%$ & J/A & $41.9 \%$ \\
\hline libpcr & $(10.09 \%)$ & $17.1 \%$ & N/A & N/A & $25.0 \%$ & $16.9 \%$ & $10.0 \%$ & $30.0 \%$ & $7.7 \%$ & N/A & $33.3 \%$ & N/A & $50.0 \%$ & $0.0 \%$ & $\mathrm{~N} / \mathrm{A}$ & $21.4 \%$ \\
\hline libcr & $(6.42 \%)$ & $20.2 \%$ & N/A & N/A & $25.0 \%$ & $20.7 \%$ & N/A & $0.0 \%$ & $21.4 \%$ & N/A & N/A & N/A & N/A & N/A & N/A & $23.1 \%$ \\
\hline libcrypto & $(83.56 \%)$ & $93.1 \%$ & N/A & $0.0 \%$ & $92.0 \%$ & $93.5 \%$ & $86.7 \%$ & $91.0 \%$ & $93.4 \%$ & $100.0 \%$ & $96.1 \%$ & $97.1 \%$ & $82.4 \%$ & $72.5 \%$ & N/A & $94.4 \%$ \\
\hline
\end{tabular}

The percentages correspond to removed gadgets (thinned vs. vanilla). Entries marked with "N/A" indicate absence of certain gadget classes in vanilla.

Table 4 reports the results of our analysis. Suite corresponds to the different applications used, along with their thinned libraries; the numbers in parentheses indicate code reduction. Total reports the overall reduction of the gadgets (thinned vs. vanilla) in each library, whereas the rest of the columns (Stack-NOP) present the reduction of certain gadget classes. For the different gadget types, we used the semantic definitions of Snow et al. [83], with additional (sub)categories for precision. Notably, and regarding AT functions, our analysis eliminates $45.19 \%$, $57.86 \%, 57.75 \%$, and $36.60 \%$ of AT functions in the four tested applications suites (i.e., Coreutils, SPEC, Nginx, and MySQL); $49.08 \%$ when we combine all of them.

All in all, the reduction of the CFI-resistant gadgets is analogous to the achieved code reduction, but, on average, the gadget reduction rate(s) are higher, suggesting that Nibbler can increase considerably the precision of backward-edge CFI schemes [107, 108]. More importantly, Nibbler can eliminate certain gadget classes in various libraries (100\% reduction), such as Load Reg. (load a register from another register or the stack), Memory (memory 
Table 5. Gadget Reduction Results

\begin{tabular}{|c|c|c|c|c|c|c|c|c|c|c|c|c|c|c|c|c|}
\hline \multirow{2}{*}{\multicolumn{2}{|c|}{ uite }} & \multirow{3}{*}{ Total } & \multicolumn{14}{|c|}{ Gadget Type } \\
\hline & & & \multicolumn{2}{|c|}{ Stack } & \multicolumn{2}{|c|}{ Load Reg. } & \multicolumn{2}{|c|}{ Memory } & \multicolumn{2}{|c|}{ Arithmetic } & \multicolumn{2}{|c|}{ Logic } & \multicolumn{2}{|c|}{ Branch } & \multirow[t]{2}{*}{ Syscall } & \multirow[t]{2}{*}{ NOP } \\
\hline & & & Pivot & Lift & Reg. & Stack & Load & Store & Reg. & Mem. & Reg. & Mem. & jmp & call & & \\
\hline \multicolumn{2}{|c|}{ SPEC } & & & & & & & & & & & & & & & \\
\hline libpthread & $2.79 \%)$ & $5.1 \%$ & $100.0 \%$ & $95.9 \%$ & $97.8 \%$ & $99.1 \%$ & $100.0 \%$ & $90.2 \%$ & $93.1 \%$ & $96.1 \%$ & $93.3 \%$ & $99.2 \%$ & $96.2 \%$ & $80.0 \%$ & $96.5 \%$ & $95.8 \%$ \\
\hline libm & $(39.76 \%)$ & $8.4 \%$ & $28.6 \%$ & $44.3 \%$ & $53.5 \%$ & $30.5 \%$ & $75.6 \%$ & $39.4 \%$ & $54.3 \%$ & $47.3 \%$ & $72.2 \%$ & $44.9 \%$ & $45.9 \%$ & $55.9 \%$ & $4 \%$ & $40.8 \%$ \\
\hline libc & $(42.01 \%)$ & $51.8 \%$ & $68.9 \%$ & $30.2 \%$ & $63.5 \%$ & $68.8 \%$ & $38.3 \%$ & $39.5 \%$ & $53.4 \%$ & $47.1 \%$ & $59.7 \%$ & $56.8 \%$ & $41.0 \%$ & $57.8 \%$ & $76.6 \%$ & $48.9 \%$ \\
\hline libgcc & $(72.35 \%)$ & $75.8 \%$ & $55.4 \%$ & $84.9 \%$ & $62.9 \%$ & $53.7 \%$ & $32.0 \%$ & $75.5 \%$ & $83.9 \%$ & $74.8 \%$ & $91.0 \%$ & $74.0 \%$ & $86.2 \%$ & $14.3 \%$ & $.0 \%$ & $77.7 \%$ \\
\hline libstdc++ & $(48.88 \%)$ & $63.9 \%$ & $67.4 \%$ & $46.2 \%$ & $60.4 \%$ & $67.5 \%$ & $51.1 \%$ & $69.3 \%$ & $72.5 \%$ & $68.5 \%$ & $68.2 \%$ & $72.9 \%$ & $56.5 \%$ & $52.9 \%$ & $50.0 \%$ & $68.2 \%$ \\
\hline \multicolumn{2}{|c|}{ Coreutils } & & & & & & & & & & & & & & & \\
\hline ppth & 4\%) & $50.4 \%$ & $51.5 \%$ & $46.5 \%$ & $53.1 \%$ & $50.1 \%$ & $44.1 \%$ & $59.8 \%$ & $44.6 \%$ & $49.9 \%$ & $56.9 \%$ & $43.2 \%$ & $48.8 \%$ & $0.0 \%$ & $44.2 \%$ & $55.2 \%$ \\
\hline bdl & $(75.41 \%)$ & $85.5 \%$ & $100.0 \%$ & $100.0 \%$ & $92.3 \%$ & $88.9 \%$ & $100.0 \%$ & $90.0 \%$ & $85.2 \%$ & $93.3 \%$ & $91.3 \%$ & $50.0 \%$ & $76.9 \%$ & $80.0 \%$ & /A & $77.3 \%$ \\
\hline libselinux & $(53.13 \%)$ & $67.3 \%$ & $64.7 \%$ & $52.9 \%$ & $62.0 \%$ & $63.2 \%$ & $80.7 \%$ & $84.7 \%$ & $65.2 \%$ & $64.2 \%$ & $84.1 \%$ & $79.9 \%$ & $62.2 \%$ & $91.8 \%$ & J/A & $63.8 \%$ \\
\hline libacl & $(69.99 \%)$ & $70.4 \%$ & $60.0 \%$ & $74.4 \%$ & $80.0 \%$ & $65.9 \%$ & $69.2 \%$ & $57.1 \%$ & $52.7 \%$ & $86.5 \%$ & $78.2 \%$ & $73.5 \%$ & $73.9 \%$ & $91.4 \%$ & N/A & $67.6 \%$ \\
\hline prt & $(71.64 \%)$ & $70.3 \%$ & $80.8 \%$ & $85.2 \%$ & $70.3 \%$ & $77.2 \%$ & $40.0 \%$ & $79.7 \%$ & $62.6 \%$ & $59.7 \%$ & $66.7 \%$ & $73.7 \%$ & $81.0 \%$ & $50.0 \%$ & $66.7 \%$ & $69.3 \%$ \\
\hline libpcre & $(10.15 \%)$ & $12.1 \%$ & $22.6 \%$ & $10.2 \%$ & $15.2 \%$ & $7.9 \%$ & $16.7 \%$ & $21.2 \%$ & $11.5 \%$ & $11.3 \%$ & $22.6 \%$ & $12.9 \%$ & $8.1 \%$ & $12.8 \%$ & $.0 \%$ & $16.0 \%$ \\
\hline libc & $(28.28 \%)$ & $38.6 \%$ & $45.0 \%$ & $21.8 \%$ & $49.0 \%$ & $45.9 \%$ & $33.4 \%$ & $31.0 \%$ & $44.5 \%$ & $35.2 \%$ & $47.3 \%$ & $43.7 \%$ & $28.5 \%$ & $50.1 \%$ & $1 \%$ & $36.2 \%$ \\
\hline batt & 7\%) & $32.4 \%$ & $68.4 \%$ & $50.0 \%$ & $10.0 \%$ & $71.1 \%$ & $0.0 \%$ & $22.2 \%$ & $37.9 \%$ & $25.7 \%$ & $18.2 \%$ & $25.0 \%$ & $6.7 \%$ & $0.0 \%$ & J/A & $46.1 \%$ \\
\hline ggn & $(46$ & $7.3 \%$ & $61.9 \%$ & $49.2 \%$ & $65.7 \%$ & $60.0 \%$ & $42.6 \%$ & $48.3 \%$ & $66.0 \%$ & $55.6 \%$ & $55.0 \%$ & $63.2 \%$ & $2.2 \%$ & $48.2 \%$ & J/A & $55.1 \%$ \\
\hline libgcc & $(92$ & $8.2 \%$ & $95.9 \%$ & $99.6 \%$ & $99.0 \%$ & $99.0 \%$ & $92.8 \%$ & $98.0 \%$ & $97.6 \%$ & 95 & $\%$ & $98.0 \%$ & $7 \%$ & $85.7 \%$ & $\%$ & $97.5 \%$ \\
\hline libcap & (49. & $56.8 \%$ & $62.5 \%$ & $0.0 \%$ & $75.9 \%$ & $54.1 \%$ & $100.0 \%$ & $78.6 \%$ & $0.8 \%$ & $29.2 \%$ & $50.0 \%$ & $50.0 \%$ & $54.5 \%$ & $20.0 \%$ & & $51.9 \%$ \\
\hline \multicolumn{2}{|c|}{ MySQL } & & & & & & & & & & & & & & & \\
\hline bm & $\%)$ & $9.9 \%$ & $29.9 \%$ & $45.4 \%$ & $54.7 \%$ & $32.4 \%$ & $75.6 \%$ & $41.8 \%$ & $55.8 \%$ & $47.9 \%$ & $71.9 \%$ & $46.4 \%$ & $51.6 \%$ & $55.9 \%$ & $.4 \%$ & $42.4 \%$ \\
\hline libpthread & 3\%) & $4.4 \%$ & $47.0 \%$ & $37.8 \%$ & $48.6 \%$ & $45.6 \%$ & $44.1 \%$ & $50.4 \%$ & $39.9 \%$ & $41.4 \%$ & $50.5 \%$ & $43.9 \%$ & $38.7 \%$ & $0.0 \%$ & $40.7 \%$ & $46.6 \%$ \\
\hline libz & $(29.37 \%)$ & $47.8 \%$ & $38.9 \%$ & $22.6 \%$ & $63.6 \%$ & $40.7 \%$ & $79.8 \%$ & $57.8 \%$ & $57.3 \%$ & $43.3 \%$ & $66.0 \%$ & $27.3 \%$ & $39.6 \%$ & $59.3 \%$ & N/A & $51.6 \%$ \\
\hline libc & $(34.23 \%)$ & $42.5 \%$ & $55.5 \%$ & $26.1 \%$ & $53.2 \%$ & $54.3 \%$ & $30.4 \%$ & $30.7 \%$ & $45.3 \%$ & $38.0 \%$ & $48.9 \%$ & $45.3 \%$ & $34.3 \%$ & $44.5 \%$ & $57.0 \%$ & $41.0 \%$ \\
\hline libgcc & $(56.10 \%)$ & $61.1 \%$ & $44.6 \%$ & $59.9 \%$ & $60.9 \%$ & $40.1 \%$ & $22.7 \%$ & $46.9 \%$ & $72.1 \%$ & $53.6 \%$ & $81.2 \%$ & $62.0 \%$ & $73.3 \%$ & $14.3 \%$ & $0.0 \%$ & $63.7 \%$ \\
\hline libstdc++ & $(45.04 \%)$ & $59.0 \%$ & $60.5 \%$ & $39.1 \%$ & $54.4 \%$ & $62.2 \%$ & $46.9 \%$ & $64.8 \%$ & $68.9 \%$ & $63.4 \%$ & $64.4 \%$ & $64.9 \%$ & $53.2 \%$ & $49.7 \%$ & $50.0 \%$ & $63.6 \%$ \\
\hline libdl & $(26.70 \%)$ & $38.6 \%$ & $0.0 \%$ & $0.0 \%$ & $35.9 \%$ & $27.8 \%$ & $78.3 \%$ & $43.3 \%$ & $37.0 \%$ & $68.3 \%$ & $56.5 \%$ & $0.0 \%$ & $46.2 \%$ & $33.3 \%$ & N/A & $33.3 \%$ \\
\hline libcrypt & $0 \%)$ & $7.3 \%$ & $14.3 \%$ & $5.0 \%$ & $6.2 \%$ & $9.5 \%$ & $25.0 \%$ & $0.0 \%$ & $10.9 \%$ & $4.0 \%$ & $0.0 \%$ & $8.7 \%$ & $0.0 \%$ & $0.0 \%$ & $0.0 \%$ & $5.7 \%$ \\
\hline \multicolumn{2}{|c|}{ in $x$} & & & & & & & & & & & & & & & \\
\hline ppthread & 5\%) & $9.3 \%$ & $48.5 \%$ & $49.4 \%$ & $47.2 \%$ & $49.7 \%$ & $42.6 \%$ & $54.1 \%$ & $43.2 \%$ & $51.5 \%$ & $55.1 \%$ & $47.0 \%$ & $52.5 \%$ & $0.0 \%$ & $43.0 \%$ & $53.5 \%$ \\
\hline libz & $(60.91 \%)$ & $.5 \%$ & $63.9 \%$ & $53.8 \%$ & $80.3 \%$ & $60.7 \%$ & $87.9 \%$ & $67.5 \%$ & $81.1 \%$ & $62.2 \%$ & $78.7 \%$ & $78.2 \%$ & $66.7 \%$ & $70.4 \%$ & N/A & $74.3 \%$ \\
\hline libc & $(40.78 \%)$ & $49.2 \%$ & $64.0 \%$ & $32.3 \%$ & $58.2 \%$ & $64.0 \%$ & $38.1 \%$ & $36.1 \%$ & $51.9 \%$ & $44.5 \%$ & $56.5 \%$ & $58.0 \%$ & $39.3 \%$ & $58.0 \%$ & $59.4 \%$ & $46.7 \%$ \\
\hline libdl & $(26.70 \%)$ & $38.6 \%$ & $0.0 \%$ & $0.0 \%$ & $35.9 \%$ & $27.8 \%$ & $78.3 \%$ & $43.3 \%$ & $37.0 \%$ & $68.3 \%$ & $56.5 \%$ & $0.0 \%$ & $46.2 \%$ & $33.3 \%$ & N/A & $33.3 \%$ \\
\hline libpcre & $(10.09 \%)$ & $11.5 \%$ & $22.6 \%$ & $10.2 \%$ & $14.2 \%$ & $7.9 \%$ & $16.2 \%$ & $17.6 \%$ & $11.1 \%$ & $10.3 \%$ & $19.2 \%$ & $12.2 \%$ & $7.9 \%$ & $12.8 \%$ & $0.0 \%$ & $15.0 \%$ \\
\hline libcrypt & $(6.42 \%)$ & $11.1 \%$ & $25.0 \%$ & $7.1 \%$ & $6.2 \%$ & $13.9 \%$ & $25.0 \%$ & $0.0 \%$ & $10.9 \%$ & $6.0 \%$ & $25.0 \%$ & $8.7 \%$ & $0.0 \%$ & $0.0 \%$ & $0.0 \%$ & $10.6 \%$ \\
\hline bcrypto & $(83.56 \%)$ & $.2 \%$ & $90.8 \%$ & $0.7 \%$ & $87.7 \%$ & $92.3 \%$ & $87.1 \%$ & $85.8 \%$ & $90.5 \%$ & $79.3 \%$ & $87.6 \%$ & $88.3 \%$ & $90.3 \%$ & $83.3 \%$ & $1.7 \%$ & $89.3 \%$ \\
\hline
\end{tabular}

The percentages correspond to removed gadgets (thinned vs. vanilla). Entries marked with "N/A" indicate absence of certain gadget classes in vanilla.

$\mathrm{read} /$ write), Arithmetic+Mem. (arithmetic computations with memory operands), Logic (logic computations), and Branch (indirect JMP/CALL), even on applications that experience moderate code reduction rates, such as Coreutils, as shown in Table 4 (underlined entries).

Gadget reduction. When CFI is not present, many more gadgets are available to attackers, so Nibbler can achieve complete gadget elimination in less cases. Table 5 summarizes our findings regarding conventional gadgets. Similar to the previous case, the reduction of gadgets is analogous to the achieved code reduction. Again, Nibbler seems to be very effective on certain gadget classes, like Branch, where the achieved gadget reduction is (on average) a bit higher than the respective code reduction. Similarly, Stack Pivot and Stack Lift gadgets, in certain real-world applications, such as Coreutils and MySQL, are reduced considerably (again on average) or eliminated completely (e.g., libdl in Coreutils). Our results indicate that although Nibbler does not entirely protect against code reuse, it raises the bar significantly for an attacker that tries to automatically stitch together code snippets 


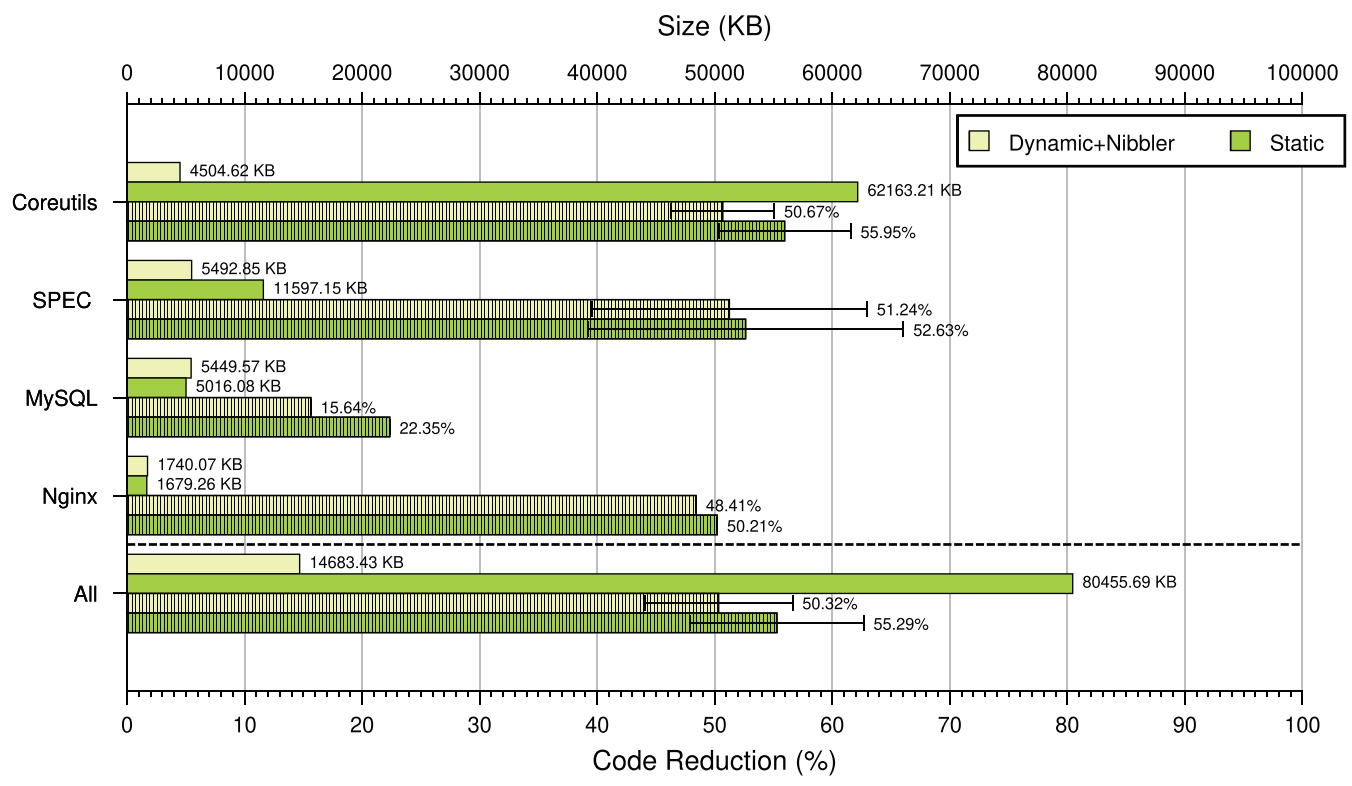

Fig. 5. Comparison of total app. size (in KB; binaries + required libraries), when using Nibbler with dynamically linked binaries (shared libraries) vs. statically linked binaries (library code is duplicated)-top $\mathrm{x}$-axis. The hatched bars correspond to average code reduction and standard deviation of total app. size across a set-bottom $\mathrm{x}$-axis.

to mount a ROP/JOP/COP attack $[22,76,77,79]$; and it achieves this with practically zero runtime performance overhead.

Effect on real-world exploits. We evaluated Nibbler against pre-compiled, real-world core-reuse exploits. Specifically, we replicated: (1) a ROP-based exploit against Nginx (CVE-2013-2028 ${ }^{2}$ ), (2) a ROP/ret2libc-based exploit against mcrypt (CVE-2012-4409 ${ }^{3}$ ), and (3) a ret2libc-based exploit against Tinyproxy/glibc (CVE-2015-7547 ${ }^{4}$ ). Next, we nibbled the libraries of the applications and re-tested the exploits. In all cases, Nibbler managed to stop the attack, by removing gadgets, required by the exploit(s), or whole (libc) functions. Note that the attacker can easily modify their exploits to use gadgets, or whole functions, from the residual code. The purpose of this experiment, however, was to demonstrate that Nibbler can thwart canned exploits with no additional runtime overhead.

5.1.4 Comparison with Static Linking. Shared libraries have numerous advantages over static ones in real-world deployments. Nevertheless, static linking, especially with the addition of link-time optimization (LTO) [56], has the potential to eliminate even more code. We compare Nibbler with static linking to highlight its debloating capabilities, as well as the large overhead associated with static linking. We statically linked all applications in our set and compared the total size of the statically linked applications against the dynamically linked ones, with all their required libraries, after we have applied Nibbler. Figure 5 shows the total size of each application set. On average, static linking reduces the size of the application by $50.32 \%$, while Nibbler by $55.29 \%$, indicating that our code-elimination techniques approximate what can be achieved through recompilation and

\footnotetext{
${ }^{2}$ https://github.com/danghvu/nginx-1.4.0.

${ }^{3}$ https://www.exploit-db.com/exploits/22928/.

${ }^{4}$ https://researchcenter.paloaltonetworks.com/2016/05/how-cve-2015-7547-glibc-getaddrinfo-can-bypass-aslr/.
} 
Table 6. Per-library Debloating in Nginx with Nibbler, Static Linking, and LTO

\begin{tabular}{lr|r|r|r}
\hline \multicolumn{4}{c}{ Code in Scope } \\
\hline \multicolumn{4}{c}{ \# of Functions (Size in KB) } \\
& Dynamic & \multicolumn{1}{c}{ Nibbler } & Static & Static+LTO \\
\hline libcrypto & 5,085 & 317 & 33 & 33 \\
libc & $(1,155.25)$ & $(105.76)$ & $(22.14)$ & $(22.14)$ \\
& 2,889 & 921 & 1,025 & 1,025 \\
libpthread & $(1,172.84)$ & $(683.24)$ & $(697.08)$ & $(697.08)$ \\
& 297 & 142 & 55 & 55 \\
libz & $(49.43)$ & $(25.69)$ & $(6.01)$ & $(6.01)$ \\
& 140 & 46 & 66 & 41 \\
libpcre & $(84.55)$ & $(31.83)$ & $(40.01)$ & $(28.35)$ \\
& 74 & 45 & 48 & 22 \\
libcrypt & $(158.82)$ & $(115.56)$ & $(122.57)$ & $(108.49)$ \\
& 39 & 28 & 37 & 37 \\
libdl & $(20.45)$ & $(19.29)$ & $(30.38)$ & $(30.38)$ \\
& 24 & 16 & 9 & 9 \\
Total (Lib.) & $(2.54)$ & $(1.69)$ & $(0.06)$ & $(0.06)$ \\
& $(2,643.89)$ & $(990.07)$ & $(911.24)$ & $(892.51)$ \\
\hline
\end{tabular}

static linking. However, if we look at the total code in the system, the first (statically linked) takes $78.57 \mathrm{MB}$, while the latter (nibbled) only 14.34 MB.

Nibbler vs. LTO. LTO corresponds to inter-procedural optimizations, performed during linking, which may eliminate even more code. To measure its effect, we built Nginx with LLVM/Clang (its LTO implementation is more mature than GCC's). We found that LTO does not significantly eliminate code when compared to static linking without LTO. Table 6 summarizes the debloating effects on library code. We notice that LTO does not significantly eliminate code, as code attributed to libraries is reduced only by $2.1 \%$, compared to conventional static linking. Interestingly, certain libraries may require additional functions/code when they are built statically, allowing Nibbler to achieve better results than static linking with LTO in such cases (see libc and libcrypt in Table 6). In general, both types of static linkage result in $\approx 10 \%$ less library code than Nibbler's thinned libraries, indicating that our code-elimination techniques approximate the "optimal" reduction rate(s) sufficiently.

\subsection{Large-scale Debloating}

To better understand code bloat at large, we ran Nibbler on (almost) all C/C++ applications in Debian sid, with the goal of reporting on unused code across a complete Linux distribution. We chose Debian sid (i.e., the development distribution of Debian) due to its high availability of debug symbol packages [99].

At the time of writing, Debian sid contained over 50K x86-64 packages, distributed over three major repositories: main, contrib, and nonfree. The first step in our analysis involved identifying the list of packages that contained executable $\mathrm{C} / \mathrm{C}++$ binaries. To build a list of candidate packages, we excluded packages that did not contain executable code by definition, including: documentation packages $(*-$ doc); development headers $(*-\mathrm{dev})$; debug symbols (*-dbg, $*$-dbgsym); metapackages and virtual packages; architecture-agnostic packages (architecture type "all”), which cannot include x86-64 binaries; kernel packages; and packages that contain only 
shared libraries. Note that shared libraries and other excluded packages can be installed during processing as dependencies of candidate packages.

We performed our analysis using the Egalito-based implementation of Nibbler, on a single host, armed with an 8-core AMD Ryzen 2700X 3.7 GHz CPU with 64 GB of RAM, running Arch Linux (kernel v5.2). For each candidate package, we install the package itself, its dependencies, and all required debug symbols and run Nibbler on each binary included. The median time to process each binary is around $20 \mathrm{~s}$, with $90 \%$ of binaries completing in under $200 \mathrm{~s}$-this is a substantial improvement over our older, Python-based prototype (see Section 4), which required more than $60 \mathrm{~s}$ per binary [2]. In total, we processed 34,537 binaries across 8,641 packages, of which 30,631 $(\approx 91.7 \%)$ could be analyzed successfully (the rest $8.3 \%$ corresponds to binaries with missing symbols, non-C/C++ code, etc.). Across the set of all the successfully analyzed binaries, we observed 5,295 unique dynamic shared libraries.

Bloat in Popular Libraries. For each binary, Nibbler reports the set of libraries used by the application, the total set of functions in each library, and the set of functions that are unused and can be removed. We aggregate these results across all the $\approx 30 \mathrm{~K}$ considered binaries to determine and characterize code bloat at large (i.e., across the whole Debian distribution).

Figure 6 shows the distribution of the amount of code removed (x-axis) for the top 20 libraries observed (yaxis) as a percentage of their total size. (Shared libraries are ranked based on the number of packages they link with.) With one exception, all the binaries we analyzed (i.e., 30,631) link-with libc; the exception is the binary mtcp_restart, an executable with no shared library dependencies.

For libc, we observe that the median amount of code removed, per binary, is $\approx 35 \%$, with most binaries individually not requiring $20 \%-42 \%$ of its code. The next most popular libraries are libpthread and libnss_files, used by $\approx 20 \mathrm{~K}$ and $\approx 19 \mathrm{~K}$ binaries, respectively, demonstrating much wider distributions (i.e., $27 \%-76 \%$ and $47 \%-$ $99 \%$ ), similarly to libresolv and libstd++ (37\%-99\%, 52\%-94\%). In contrast, libnss_resolve ( $\approx 9 \mathrm{~K} \%$ binaries) has a very tight distribution with a median of only $\approx 5 \%$ of code removed, owing to the relatively small number of functions it exports and their tight coupling (i.e., all variations of gethostbyname and gethostbyaddr). libm, libdl, librt, and libbsd also exhibit tight distributions, with medians of $\approx 54 \%, \approx 29 \%, \approx 97 \%$, and $\approx 99 \%$ of code removed, respectively.

Beyond per-binary metrics, we can gain further insights by considering the union of all functions required across all binaries in the distribution, which identifies code not used by (almost) any binary in the system. Accordingly, the union amount of code to remove is always lower than the median reported in Figure 6. For libc, we observe that $\approx 5 \%$ of its code is unused across all binaries. As another example, the popular NSS [53] libraries libnss_dns and libnss_db have nearly $50 \%$ unused code across all binaries; likewise, libxcb and libstd++ exhibit $\approx 46 \%$ and $\approx 38 \%$ unused code across all binaries.

Last, Figures 7 and 8 illustrate the effect of Nibbler on popular cryptographic, and X11, libraries, across the whole Debian distribution. More importantly, our results indicate that Nibbler can be used to replace the 5,295 unique dynamic shared libraries, in Debian sid, with their "unionized" equivalents, without affecting the functionality of any binary (out of the 30,631 analyzed), while removing $\approx 420 \mathrm{MB}(\approx 1.6 \mathrm{M}$ functions) of code in total.

\subsection{Contained Debloating}

To assess the amount of code bloat in commonly distributed sets of Linux binaries, we used Nibbler to examine packages from popular container images published in the Docker Hub repository [29]. Rather than analyzing binaries in each image directly, we leverage our existing dataset and infrastructure for processing Debian sid packages (Section 5.2) to provide an equivalent analysis. Specifically, we used the container images to identify sets of binaries to consider from our Debian sid dataset. By examining the union of library functions removed across all binaries in each set, we can we can quantify the total set of unused code across all libraries in the published container image.

Digital Threats: Research and Practice, Vol. 1, No. 4, Article 19. Publication date: December 2020. 


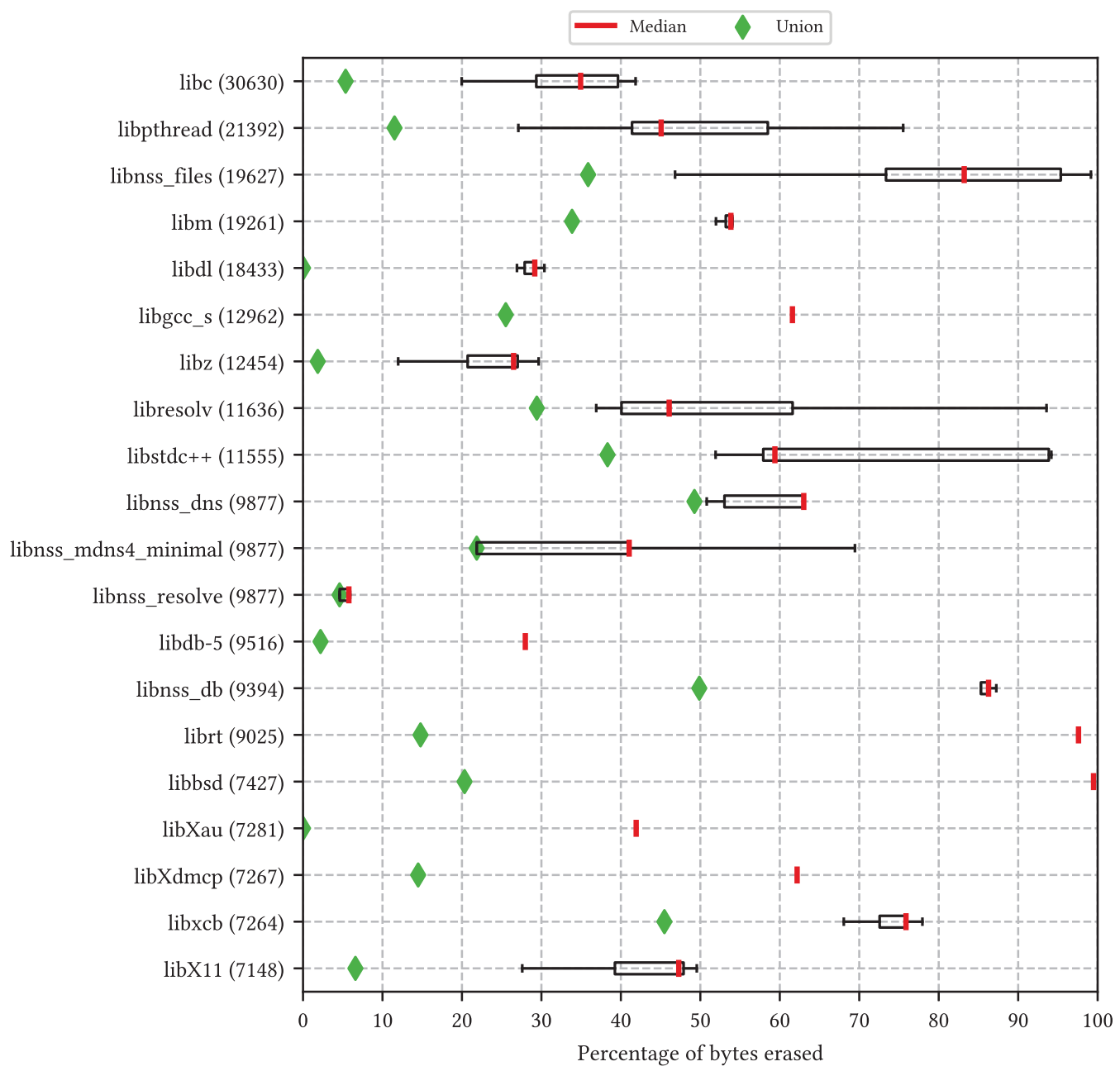

Fig. 6. Unused code (x-axis) in the 20 most popular libraries (y-axis) in Debian sid. Numbers in parentheses indicate the number of binaries (out of the 30,631 analyzed) using the respective library.

We selected nine official Docker images for popular $\mathrm{C} / \mathrm{C}++$ applications, each with $>10 \mathrm{M}$ downloads, on Docker Hub. To simplify the process of translating packages in the container's base distribution to Debian package names, we selected container images that were built from distributions using the apt package manager. For each container image, we mapped each binary to its providing package using dpkg and then looked up the equivalent sid package in our dataset. Across all images, we considered a total of 255 unique packages. Mappings for all but 11 packages could be resolved automatically-we manually resolved the remainder, which differed only by version numbers (e.g., mariadb-server-10.3 vs. mariadb-server-10.4). While this mapping between Linux distributions does not provide an exact representation of the unused code included in the container images, it does provide a tight approximation of code bloat across a commonly installed set of packages in popular Docker images.

Table 7 shows the union amount of code removed across all libraries in each container image. Every image used roughly 300 binaries and less than 100 shared libraries. Across all images, we observe that an average of $23.2 \%$ of code (in terms of by bytes) is unused. All images exhibited roughly the same amount of bloat, with 


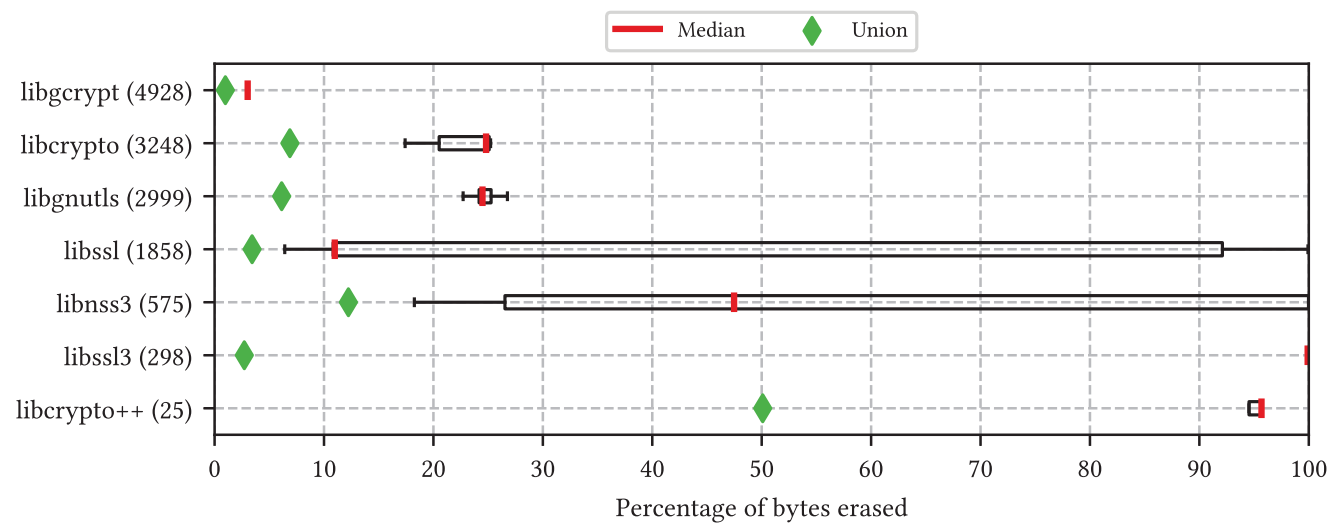

Fig. 7. Unused code (x-axis) in cryptographic libraries in Debian sid. Libraries (y-axis) used by $\leq 20$ packages are excluded. Numbers in parentheses indicate the number of binaries (out of the 30,631 analyzed) using the respective library.

Table 7. Erasure for Docker Images (Using Packages from Debian sid)

\begin{tabular}{|c|c|c|c|c|c|c|c|c|}
\hline \multirow[b]{2}{*}{ Image } & \multirow[b]{2}{*}{ Bins } & \multirow[b]{2}{*}{ Libs } & \multicolumn{3}{|c|}{ Bytes (KB) } & \multicolumn{3}{|c|}{ Functions } \\
\hline & & & Total & Erased & $\%$ & Total & Erased & $\%$ \\
\hline haproxy & 293 & 62 & $15,247.06$ & $3,565.83$ & 23.39 & 46,822 & 17,000 & 36.31 \\
\hline influxdb & 309 & 83 & $16,778.82$ & $3,397.27$ & 20.25 & 52,240 & 16,822 & 32.20 \\
\hline mariadb & 394 & 84 & $17,466.90$ & $3,790.69$ & 21.70 & 53,231 & 18,364 & 34.50 \\
\hline memcached & 292 & 61 & $13,683.17$ & $3,393.47$ & 24.80 & 39,621 & 14,606 & 36.86 \\
\hline nginx & 294 & 59 & $13,425.71$ & $3,263.02$ & 24.30 & 38,531 & 13,953 & 36.21 \\
\hline postgres & 363 & 83 & $21,047.71$ & $4,808.17$ & 22.84 & 69,125 & 23,817 & 34.45 \\
\hline redis & 295 & 68 & $15,886.73$ & $3,649.33$ & 22.97 & 47,964 & 17,367 & 36.21 \\
\hline spiped & 296 & 60 & $14,828.60$ & $3,590.70$ & 24.21 & 44,949 & 17,048 & 37.93 \\
\hline ubuntu & 310 & 61 & $13,549.62$ & $3,292.48$ & 24.30 & 39,077 & 14,261 & 36.49 \\
\hline \multicolumn{3}{|c|}{ Arithmetic mean } & $15,768.26$ & $3,638.99$ & 23.20 & 47,951 & 17,026 & 35.69 \\
\hline \multicolumn{3}{|c|}{ Geometric mean } & $15,613.71$ & $3,615.18$ & 23.15 & 47,177 & 16,818 & 35.65 \\
\hline
\end{tabular}

image-wide union values within $4 \%$ of each other. The majority of this bloat is due to common system libraries used in each image, rather than application-specific libraries-we leave the analysis of comparing ancillary utilities and binaries required for the application's use-case as a future study. Interestingly, in seven out of the nine images, the library with the most code removed (>94\%) was libunistring [32], a common library for handling Unicode. Conversely, all images utilized one library that had no code removed, i.e., libdebconfclient [26], which is used by dpkg. Overall, our results demonstrate opportunities for reducing code bloat, using Nibbler, in commonly used container images.

\subsection{Reduction with Application-loaded Libraries}

Chromium (web browser; v57) is a large, complex application that performs manual library loading. To debloat it, we profile it by: (i) visiting the top sites in Alexa's "Top 500 Global Sites" list [4], exercising a broad range of functionality, such as video playback, animations, and so on; and (ii) using Chromium's comprehensive test suite [89], which includes a plethora of tests related to layout and rendering, conformance to certain web standards, UI events, and Chrome-specific APIs. During profiling the browser loaded 63 additional libraries. After 


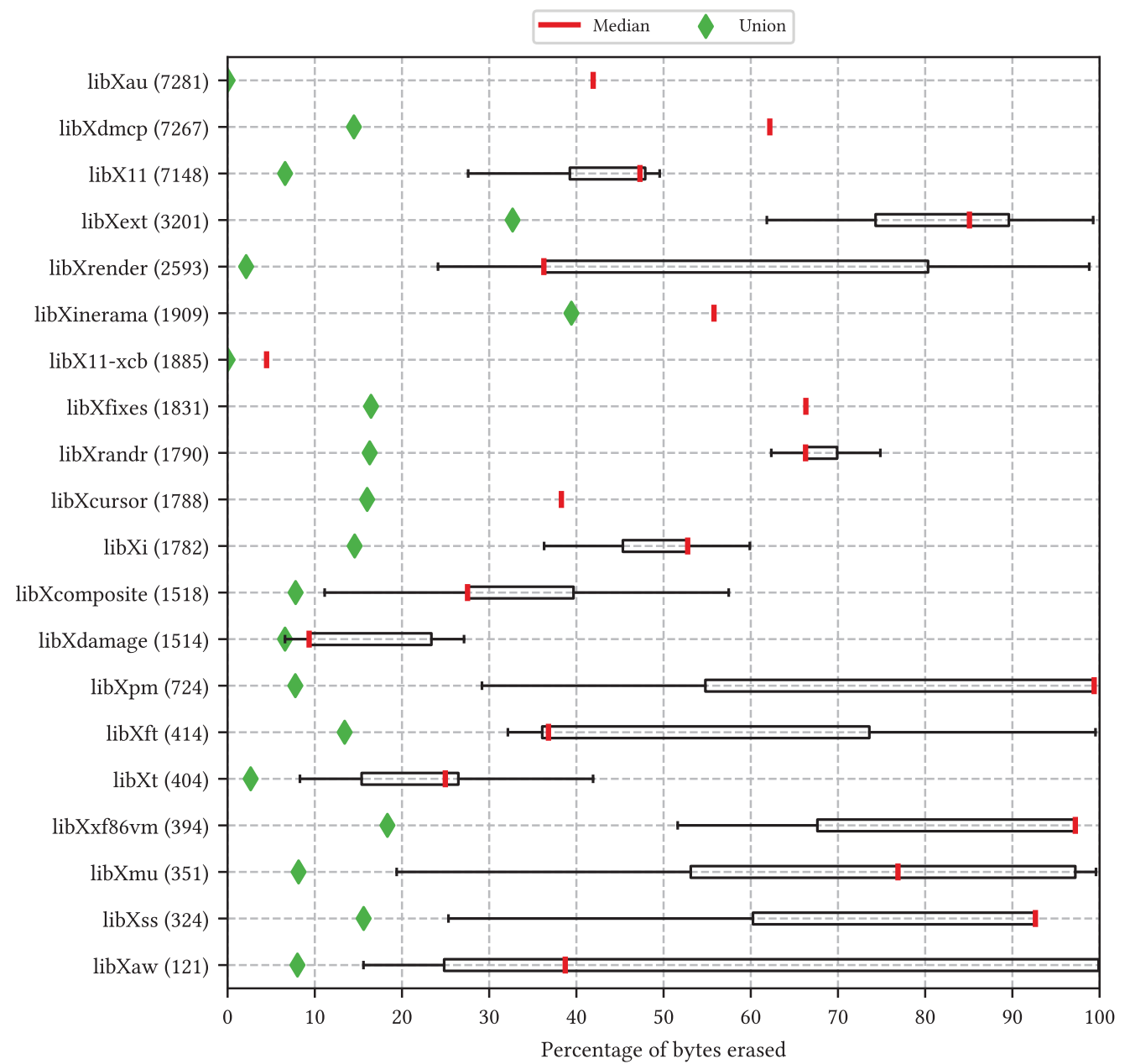

Fig. 8. Unused code (x-axis) in X11 libraries in Debian sid. Libraries (y-axis) used by $\leq 20$ packages are excluded. Numbers in parentheses indicate the number of binaries (out of the 30,631 analyzed) using the respective library.

including the used symbols in Nibbler, we included an additional 3,241 functions or approximately $1 \mathrm{MB}$ of code. Table 8 lists the 10 (out of 84) most thinned libraries and total code reduction in Chromium. Finally, we browsed the top-10 sites in Alexa's list, which did not result in new libraries being loaded. Our experiment confirms the results of previous work [70], which showed that profiling can be sufficient to debloat manually loaded libraries in certain applications.

Note, however, that by employing our value-tracking approach (see Section 3.7), Nibbler can statically resolve $\approx 89 \%$ of all dlsym() arguments and $\approx 37 \%$ of all dlopen( $)$ arguments, across the $\approx 30 \mathrm{~K}$ binaries and $\approx 5 \mathrm{~K}$ unique shared libraries in Debian sid (see Section 5.2).

\section{LIMITATIONS}

Exploit disruption. In Section 5.1.3, we tested our nibbled applications against pre-compiled, real-world codereuse exploits. In all cases, Nibbler disrupted the respective exploit; however, attackers can modify them to use other gadgets and potentially restore their capabilities. Debloating, even when combined with CFI, cannot block 
Table 8. Ten Most Debloated Libraries in Chromium

\begin{tabular}{l|rr|rr}
\hline \multirow{2}{*}{ Library } & \multicolumn{4}{|c}{ Unused Code } \\
\cline { 2 - 6 } & \multicolumn{2}{|c}{ Functions } & \multicolumn{2}{c}{ Bytes } \\
\hline libgtk & 4,886 & $(49.06 \%)$ & $994.70 \mathrm{~KB}$ & $(41.03 \%)$ \\
libxml2 & 1,260 & $(48.26 \%)$ & $423.87 \mathrm{~KB}$ & $(46.70 \%)$ \\
libc & 1,606 & $(46.93 \%)$ & $316.35 \mathrm{~KB}$ & $(26.25 \%)$ \\
libgio & 1,749 & $(38.65 \%)$ & $289.00 \mathrm{~KB}$ & $(35.13 \%)$ \\
libgnutls & 826 & $(36.26 \%)$ & $212.32 \mathrm{~KB}$ & $(27.76 \%)$ \\
libnss3 & 2,763 & $(70.56 \%)$ & $172.14 \mathrm{~KB}$ & $(18.05 \%)$ \\
libbglib & 840 & $(40.64 \%)$ & $154.70 \mathrm{~KB}$ & $(32.37 \%)$ \\
libasound & 1,053 & $(36.15 \%)$ & $152.99 \mathrm{~KB}$ & $(27.61 \%)$ \\
libm & 375 & $(61.68 \%)$ & $137.77 \mathrm{~KB}$ & $(32.00 \%)$ \\
libstdc++ & 842 & $(28.45 \%)$ & $115.45 \mathrm{~KB}$ & $(22.47 \%)$ \\
libX11 & 433 & $(22.29 \%)$ & $111.08 \mathrm{~KB}$ & $(19.95 \%)$ \\
\hline Total & 20,946 & $(34.95 \%)$ & $4,198.22 \mathrm{~KB}$ & $(25.98 \%)$ \\
\hline
\end{tabular}

all code-reuse attacks, but it does make libraries a less fertile ground for gadget harvesting. Exploits are more likely to be prevented by combining Nibbler with a system like Shuffler [101].

Attack-surface reduction. Previous work [70] suggested that debloating can reduce the attack surface by removing vulnerabilities contained in the erased library code. Nibbler performs a similar type of debloating on binary code. However, we did not reach the same conclusion. By design, both works remove code only when there is no viable execution path to that code for a given application. Consequently, any code removed is essentially unreachable code, so vulnerabilities contained within are not relevant, because they can never be triggered by external input(s). We do agree, however, that such vulnerabilities can potentially be used by multistage exploits, where later stage exploit components (ab)use vulnerabilities in unused code to escape sandboxing or further elevate privileges [21].

\section{RELATED WORK}

\subsection{Code Reduction}

Recent work from Quach et al. [70] proposes a compiler-based framework for debloating applications when source code is available, while Nibbler [2] targets binary-only software. Other differences with Nibbler include the following: (i) their approach is unable to work with (one of) the most commonly used libraries, GNU libc (glibc), which requires the GNU C compiler, while Nibbler is compiler-agnostic; (ii) their approach opts to debloat each application individually, which incurs significant memory overhead when applied to numerous applications, as it breaks the sharing of memory pages that include erased code per-application instantiation; (iii) they debloat applications at load time, which incurs a slowdown of $20 \times$, and, even though the overhead for launching one application is negligible in absolute terms, it compounds in applications that spawn others (e.g., shell scripts); and (iv) Nibbler goes beyond CFI by demonstrating one of the key benefits of debloating by integrating it with continuous code re-randomization.

CodeFreeze [62] aims to reduce the attack surface of Windows binaries by removing unused code in shared libraries (DLLs). It utilizes bounded address tracking [47] to resolve function pointers, which leads to overrestrictive CFGs. As a result, while it is more aggressive at removing code, it can erroneously remove needed functions (e.g., constructors), and it depends on whitelisting to avoid crashes. Instead, Nibbler's analysis is conservative and errs on the safe side by over-approximating. Our evaluation shows that we can correctly trim 
libraries without the need for a whitelist. More recently, BinTrimmer [73] introduced a new value range analysis technique, called signedness-agnostic strided interval, allowing for the construction of more precise CFGs (compared to CodeFreeze) to conservatively remove unused code.

Perses [85] and C-Reduce [74] are state-of-the-art program reduction tools that build upon the concept of (hierarchical) delta debugging $[60,106]$. Specifically, by specifying a program to be minimized and an arbitrary property test function, both these tools return a minimized version of the input program that is also correct with respect to the given property. Chisel [40] further improves this approach by leveraging reinforcement learning. In particular, via repeated trial and error, Chisel builds (and further rectifies) a model that determines the likelihood of a candidate (minimal) program to pass the property test. Razor [69], however, leverages application test suites, coupled with a set of related-code inference heuristics, to specialize binary applications while supporting userexpected functionality.

In antithesis to tools like the above, Nibbler does not require any high-level specification regarding the functionality of the input program/library. Our thinned libraries are guaranteed to be correct under any given input to the set of applications that uses them. Kurmus et al. [49] focus on reducing the attack surface of the Linux kernel by removing unnecessary features. Unlike Nibbler, they develop a tool-assisted approach for identifying and removing unnecessary features during the kernel's configuration phase, hence, omitting code during compilation. Ghaffarinia and Hamlen [34] introduce control-flow trimming, a concept that builds upon runtime tracing, machine learning, in-lined reference monitoring, and contextual control-flow integrity enforcement to automatically remove features from binary applications.

A series of works $[9,44,45,94]$ have focused on reducing bloat in PHP and Java programs, and the Java Virtual Machine (JVM). JRed [44] employs static analysis to extract the FCG of applications and identify and remove the bytecode that corresponds to unused classes and methods from the Java runtime. Similarly, Wagner et al. [94] propose "slimming" the JVM by removing code that does not execute frequently and dynamically fetching it from a server only when it is required. The goal is to reduce the amount of code that needs to be deployed in thin clients, such as embedded systems, by dynamically deploying what is required. Jiang et al. [45], instead of targeting the JVM, aim to cut specific features that are not needed from Java programs. Starting from a small set of methods responsible for implementing a feature, they use static analysis and backwards slicing to identify and remove all the code corresponding to the feature. While these approaches also utilize static analyses, decompiling and reconstructing the FCG of Java, or PHP, programs is less challenging than handling binary code $[9,39]$.

Landsborough et al. [50] also propose removing unused features from programs to reduce their attack surface. Their approach involves manually disabling features in binaries and a genetic algorithm that is applied in toy programs. Malecha et al. propose software winnowing [57], an approach that uses partial evaluation of function arguments during compilation to "specialize" code, eliminating some unused code in the process. In the same vein, TRIMMER [81] specializes program code, and debloats applications, by leveraging user-defined configurations, while Shredder [61] further introduces constant propagation analyses to specialize system API functions. Last, Koo et al. [48] propose the concept of configuration-based software debloating: i.e., the removal of featurespecific code, which is exclusively used only when certain configuration directives are specified/enabled. These approaches are orthogonal to Nibbler, looking at software thinning from a different perspective, while most of them (with the exception of Shredder) require access to source code.

Last, other works approach debloating from a performance angle, focusing on reducing memory consumption $[15,65,105]$. Despite the similarity in name, slim binaries [33], proposed by Franz et al., refer to programs that are represented in a way that allows their translation to multiple architectures.

\subsection{FCG Extraction}

Sound and complete extraction of the FCG from binaries is an open problem. Murphy et al. [63] perform an empirical analysis of static call-graph extractors that operate on source code or at compile time. Their findings 
indicate that there is significant variance, based on the tool, and the potential for false negatives. The latter correspond to undiscovered but existing call edges, which would be problematic for our approach, as removal of used code can be catastrophic. As existing FCG extraction methods are insufficient, we developed our own method that is complete. There are also various promising binary analysis and augmentation frameworks [6, 13, $14,28]$ that reconstruct the FCG of binaries. Even though these tools keep improving, errors are still possible per their authors, as well as other researchers [8]. As such, their analyses are not appropriate for Nibbler. Instead, the methods described in hardening works for binaries $[102,107,108]$ are more related to our approach. Unlike them, though, we introduce a novel methodology for eliminating AT functions-thereby deleting extraneous CFG edges-and reconstruct a complete FCG that also includes direct calls within and across modules.

\section{CONCLUSION}

In this article, we presented Nibbler, a system that demonstrates that debloating binary-only applications is possible and practical. We evaluated the debloating capabilities of Nibbler with real-world binaries and the SPEC CINT2006 suite, eliminating $56 \%$ and $82 \%$ of functions and code, respectively, from used libraries. Nibbler is able to correctly analyze binary software by only leveraging symbol and relocation information produced by existing compilers. In addition, we applied Nibbler on $\approx 30 \mathrm{~K} \mathrm{C} / \mathrm{C}++$ binaries and $\approx 5 \mathrm{~K}$ unique dynamic shared libraries (i.e., almost in the complete Debian sid distribution), as well as on nine official Docker images (with millions of downloads in Docker Hub), reporting findings regarding code bloat at large. Nibbler, and debloating generally, improves security of software indirectly, by benefiting defenses. Continuous code re-randomization systems get a performance boost, which we demonstrated by integrating Nibbler with Shuffler to lower overhead by $20 \%$. Lower overheads make such defenses more attractive for deployment on production systems or can be used to provide stricter security guarantees (e.g., by raising re-randomization frequency) in critical systems. Controlflow integrity defenses also benefit, because we remove code involved in allowable control-flows. Our evaluation shows that Nibbler reduces the number of gadgets reachable through returns and indirect calls by $75 \%$ and $49 \%$ on average.

Availability

Our prototype implementation is available at: https://gitlab.com/brown-ssl/libfilter.

\section{ACKNOWLEDGMENTS}

We thank the anonymous reviewers for their valuable feedback.

\section{REFERENCES}

[1] Martín Abadi, Mihai Budiu, Úlfar Erlingsson, and Jay Ligatti. 2005. Control-flow integrity. In Proceedings of the ACM CCS. 340-353.

[2] Ioannis Agadakos, Di Jin, David Williams-King, Vasileios P. Kemerlis, and Georgios Portokalidis. 2019. Nibbler: Debloating binary shared libraries. In Proceedings of the ACSAC. 70-83.

[3] Alfred V. Aho, Monica S. Lam, Ravi Sethi, and Jeffrey D. Ullman. 2006. Compilers: Principles, Techniques, and Tools (2nd ed.). Pearson Education.

[4] Alexa. 2018. The top 500 sites on the web. Retrieved from https://www.alexa.com/topsites.

[5] Jim Alves-Foss and Jia Song. 2019. Function boundary detection in stripped binaries. In Proceedings of the ACSAC. 84-96.

[6] Kapil Anand, Matthew Smithson, Khaled Elwazeer, Aparna Kotha, Jim Gruen, Nathan Giles, and Rajeev Barua. 2013. A compiler-level intermediate representation based binary analysis and rewriting system. In Proceedings of the EuroSys. 295-308.

[7] Starr Andersen and Vincent Abella. 2004. Changes to Functionality in Microsoft Windows XP Service Pack 2, Part 3: Memory Protection Technologies, Data Execution Prevention. Microsoft TechNet Library. Retrieved from http://technet.microsoft.com/en-us/library/ bb457155.aspx.

[8] Dennis Andriesse, Xi Chen, Victor van der Veen, Asia Slowinska, and Herbert Bos. 2016. An in-depth analysis of disassembly on full-scale x86/x64 binaries. In Proceedings of the USENIX SEC. 583-600.

Digital Threats: Research and Practice, Vol. 1, No. 4, Article 19. Publication date: December 2020. 
[9] Babak Amin Azad, Pierre Laperdrix, and Nick Nikiforakis. 2019. Less is more: Quantifying the security benefits of debloating web applications. In Proceedings of the USENIX SEC. 1697-1714.

[10] Eli Bendersky. 2020. pyelftools-Parsing ELF and DWARF in Python. Retrieved from https://github.com/eliben/pyelftools.

[11] David Bigelow, Thomas Hobson, Robert Rudd, William Streilein, and Hamed Okhravi. 2015. Timely rerandomization for mitigating memory disclosures. In Proceedings of the ACM CCS. 268-279.

[12] Andrea Bittau, Adam Belay, Ali Mashtizadeh, David Mazières, and Dan Boneh. 2014. Hacking blind. In Proceedings of the IEEE S\&P. $227-242$.

[13] David Brumley, Ivan Jager, Thanassis Avgerinos, and Edward J. Schwartz. 2011. BAP: A binary analysis platform. In Proceedings of the CAV. 463-469.

[14] David Brumley, JongHyup Lee, Edward J. Schwartz, and Maverick Woo. 2013. Native x86 decompilation using semantics-preserving structural analysis and iterative control-flow structuring. In Proceedings of the USENIX SEC. 353-368.

[15] Yingyi Bu, Vinayak Borkar, Guoqing Xu, and Michael J. Carey. 2013. A bloat-aware design for big data applications. In Proceedings of the ISMM. 119-130.

[16] Amat Cama. 2014. Tool to generate ROP gadgets for ARM, AARCH64, x86, MIPS, PPC, RISCV, SH4 and SPARC. Retrieved from https://github.com/acama/xrop.

[17] Nicolas Carlini, Antonio Barresi, Mathias Payer, David Wagner, and Thomas R. Gross. 2015. Control-flow bending: On the effectiveness of control-flow integrity. In Proceedings of the USENIX SEC. 161-176.

[18] Sang Kil Cha, Thanassis Avgerinos, Alexandre Rebert, and David Brumley. 2012. Unleashing mayhem on binary code. In Proceedings of the IEEE S\&P. 380-394.

[19] Stephen Checkoway, Lucas Davi, Alexandra Dmitrienko, Ahmad-Reza Sadeghi, Hovav Shacham, and Marcel Winandy. 2010. Returnoriented programming without returns. In Proceedings of the ACM CCS. 559-572.

[20] Xi Chen, Herbert Bos, and Cristiano Giuffrida. 2017. CodeArmor: Virtualizing the code space to counter disclosure attacks. In Proceedings of the IEEE EuroS\&P. 514-529.

[21] Chromium Blog. 2012. A Tale of Two Pwnies. Retrieved from https://blog.chromium.org/2012/05/tale-of-two-pwnies-part-1.html.

[22] Corelan. 2011. Corelan Repository for mona.py. Retrieved from https://github.com/corelan/mona.

[23] Stephen Crane, Per Larsen, Stefan Brunthaler, and Michael Franz. 2013. Booby trapping software. In Proceedings of the NSPW. 95-106.

[24] National Vulnerability Database. 2019. BlueKeep Vulnerability (CVE-2019-0708). NIST. Retrieved from https://nvd.nist.gov/vuln/ detail/CVE-2019-0708.

[25] Bruce Dawson. 2013. Symbols on Linux update: Fedora Fixes. Retrieved from https://randomascii.wordpress.com/2013/03/05/symbolson-linux-update-fedora-fixes/.

[26] Debian. 2020. Package: cdebconf. Retrieved from https://packages.debian.org/sid/cdebconf.

[27] Solar Designer. 1997. Getting around non-executable stack (and fix). BugTraq. Retrieved from https://seclists.org/bugtraq/1997/Aug/63.

[28] Sushant Dinesh, Nathan Burow, Dongyan Xu, and Mathias Payer. 2020. RetroWrite: Statically instrumenting COTS binaries for fuzzing and sanitization. In Proceedings of the IEEE S\&P. 128-142.

[29] Docker. 2020. Docker Hub. Retrieved from https://hub.docker.com.

[30] Ulrich Drepper. [n.d.]. ELF Symbol Versioning. Retrieved from https://www.akkadia.org/drepper/symbol-versioning.

[31] Isaac Evans, Fan Long, Ulziibayar Otgonbaatar, Howard Shrobe, Martin Rinard, Hamed Okhravi, and Stelios Sidiroglou-Douskos. 2015. Control Jujutsu: On the weaknesses of fine-grained control flow integrity. In Proceedings of the ACM CCS. 901-913.

[32] Free Software Foundation. 2019. libunistring. Retrieved from https://www.gnu.org/software/libunistring/.

[33] Michael Franz and Thomas Kistler. 1997. Slim binaries. Commun. ACM 40, 12 (Dec. 1997), 87-94.

[34] Masoud Ghaffarinia and Kevin W. Hamlen. 2019. Binary control-flow trimming. In Proceedings of the ACM CCS. $1009-1022$.

[35] Cristiano Giuffrida, Anton Kuijsten, and Andrew S. Tanenbaum. 2012. Enhanced operating system security through efficient and fine-grained address space randomization. In Proceedings of the USENIX SEC. 475-490.

[36] GNU Development Tools. 2020. objdump-display information from object files. Retrieved from http://man7.org/linux/man-pages/ man1/objdump.1.html.

[37] Enes Göktas, Elias Athanasopoulos, Herbert Bos, and Georgios Portokalidis. 2014. Out of control: Overcoming control-flow integrity. In Proceedings of the IEEE S\&P. 575-589.

[38] Enes Göktas, Benjamin Kollenda, Philipp Koppe, Erik Bosman, Georgios Portokalidis, Thorsten Holz, Herbert Bos, and Cristiano Giuffrida. 2018. Position-independent code reuse: On the effectiveness of ASLR in the absence of information disclosure. In Proceedings of the IEEE EuroS\&P. 227-242.

[39] James Hamilton and Sebastian Danicic. 2009. An evaluation of current Java bytecode decompilers. In Proceedings of the IEEE SCAM. 129-136.

[40] Kihong Heo, Woosuk Lee, Pardis Pashakhanloo, and Mayur Naik. 2018. Effective program debloating via reinforcement learning. In Proceedings of the ACM CCS. 380-394.

[41] Hex-Rays. 2016. The IDA Pro Disassembler and Debugger. Retrieved from https://www.hex-rays.com/products/ida/. 
[42] Patrick Horgan. 2011. Linux x86 Program Start Up. Retrieved from http://dbp-consulting.com/tutorials/debugging/ linuxProgramStartup.html.

[43] Intel. 2013. System V Application Binary Interface. Retrieved from https://software.intel.com/sites/default/files/article/402129/mpxlinux64-abi.pdf.

[44] Yufei Jiang, Dinghao Wu, and Peng Liu. 2016. JRed: Program customization and bloatware mitigation based on static analysis. In Proceedings of the IEEE COMPSAC. 12-21.

[45] Yufei Jiang, Can Zhang, Dinghao Wu, and Peng Liu. 2016. Feature-based software customization: Preliminary analysis, formalization, and methods. In Proceedings of the IEEE HASE. 122-131.

[46] JoeDog. 2017. Siege-An http load tester and benchmarking utility. Retrieved from https://github.com/JoeDog/siege.

[47] Johannes Kinder and Helmut Veith. 2010. Precise static analysis of untrusted driver binaries. In Proceedings of the FMCAD. 43-50.

[48] Hyungjoon Koo, Seyedhamed Ghavamnia, and Michalis Polychronakis. 2019. Configuration-driven software debloating. In Proceedings of the EuroSec.

[49] Anil Kurmus, Reinhard Tartler, Daniela Dorneanu, Bernhard Heinloth, Valentin Rothberg, Andreas Ruprecht, Wolfgang SchröderPreikschat, Daniel Lohmann, and Rüdiger Kapitza. 2013. Attack surface metrics and automated compile-time OS kernel tailoring. In Proceedings of the NDSS.

[50] Jason Landsborough, Stephen Harding, and Sunny Fugate. 2015. Removing the kitchen sink from software. In Proceedings of the ACM GECCO. 833-838.

[51] John R. Levine. 1999. Linkers and Loaders (1st ed.). Morgan Kaufmann Publishers Inc., San Francisco, CA.

[52] Percy Liang and Mayur Naik. 2011. Scaling abstraction refinement via pruning. In Proceedings of the ACM PLDI. 590-601.

[53] The GNU C Library. 2020. System Databases and Name Service Switch. Retrieved from https://www.gnu.org/software/libc/manual/ html_node/Name-Service-Switch.html.

[54] Linux Programmer's Manual. 2018. rtld-audit-Auditing API for the dynamic linker. Retrieved from http://man7.org/linux/man-pages/ man7/rtld-audit.7.html.

[55] Generic Part Linux Standard Base Core Specification. 2015. Exception Frames. Retrieved from https://refspecs.linuxbase.org/LSB_5.0. 0/LSB-Core-generic/LSB-Core-generic/ehframechpt.html.

[56] LLVM Project. 2018. LLVM Link Time Optimization: Design and Implementation. https://lvm.org/docs/LinkTimeOptimization.html.

[57] Gregory Malecha, Ashish Gehani, and Natarajan Shankar. 2015. Automated software winnowing. In Proceedings of the ACM SAC. 1504-1511.

[58] Microsoft. 2015. Control Flow Guard. Windows Dev Center. Retrieved from https://msdn.microsoft.com/en-us/library/windows/ desktop/mt637065(v=vs.85).aspx.

[59] Microsoft. 2017. Symbols and Symbol Files. Microsoft Developer Network. Retrieved from https://msdn.microsoft.com/en-us/library/ ff558825.aspx.

[60] Ghassan Misherghi and Zhendong Su. 2006. HDD: Hierarchical delta debugging. In Proceedings of the ICSE. 142-151.

[61] Shachee Mishra and Michalis Polychronakis. 2018. Shredder: Breaking exploits through API specialization. In Proceedings of the ACSAC. ACM, 1-16.

[62] Collin Mulliner and Matthias Neugschwandtner. 2015. Breaking Payloads with Runtime Code Stripping and Image Freezing. BHUSA. Retrieved from https://www.blackhat.com/us-15/briefings.html\#breaking-payloads-with-runtime-code-stripping-andimage-freezing.

[63] Gail C. Murphy, David Notkin, William G. Griswold, and Erica S. Lan. 1998. An empirical study of static call graph extractors. ACM Trans. Softw. Eng. Methodol. 7, 2 (Apr. 1998), 158-191.

[64] National Security Agency. 2019. Ghidra. nsa.gov. Retrieved from https://www.nsa.gov/resources/everyone/ghidra/.

[65] Khanh Nguyen and Guoqing Xu. 2013. Cachetor: Detecting cacheable data to remove bloat. In Proceedings of the ACM ESEC/FSE. $268-278$.

[66] Ben Niu and Gang Tan. 2014. Modular control-flow integrity. In Proceedings of the ACM PLDI. 577-587.

[67] Ben Niu and Gang Tan. 2015. Per-input control-flow integrity. In Proceedings of the ACM CCS. 914-926.

[68] PaX Team. 2003. Address Space Layout Randomization (ASLR). Retrieved from https://pax.grsecurity.net/docs/aslr.txt.

[69] Chenxiong Qian, Hong Hu, Mansour Alharthi, Pak Ho Chung, Taesoo Kim, and Wenke Lee. 2019. RAZOR: A framework for postdeployment software debloating. In Proceedings of the USENIX SEC. 1733-1750.

[70] Anh Quach, Aravind Prakash, and Lok Yan. 2018. Debloating software through piece-wise compilation and loading. In Proceedings of the USENIX SEC. 869-886.

[71] Nguyen Anh Quynh. 2014. Capstone: Next-gen disassembly framework. In Proceedings of the BHUSA.

[72] Ganesan Ramalingam. 1994. The undecidability of aliasing. ACM Trans. Prog. Lang. Syst. 16, 5 (1994), 1467-1471.

[73] Nilo Redini, Ruoyu Wang, Aravind Machiry, Yan Shoshitaishvili, Giovanni Vigna, and Christopher Kruegel. 2019. Bintrimmer: Towards static binary debloating through abstract interpretation. In Proceedings of the DIMVA. 482-501.

[74] John Regehr, Yang Chen, Pascal Cuoq, Eric Eide, Chucky Ellison, and Xuejun Yang. 2012. Test-case reduction for C compiler bugs. In Proceedings of the ACM PLDI. 335-346.

Digital Threats: Research and Practice, Vol. 1, No. 4, Article 19. Publication date: December 2020. 
[75] Robert Rudd, Richard Skowyra, David Bigelow, Veer Dedhia, Thomas Hobson, Stephen Crane, Christopher Liebchen, Per Larsen, Lucas Davi, Michael Franz, Ahmad-Reza Sadeghi, and Hamed Okhravi. 2017. Address oblivious code reuse: On the effectiveness of leakage resilient diversity. In Proceedings of the NDSS.

[76] Jonathan Salwan. 2011. ROPgadget-Gadgets finder and auto-roper. Retrieved from http://shell-storm.org/project/ROPgadget/.

[77] Sascha Schirra. 2014. Ropper-rop gadget finder and binary information tool. Retrieved from https://scoding.de/ropper/.

[78] Felix Schuster, Thomas Tendyck, Christopher Liebchen, Lucas Davi, Ahmad-Reza Sadeghi, and Thorsten Holz. 2015. Counterfeit object-oriented programming: On the difficulty of preventing code reuse attacks in $\mathrm{C}++$ applications. In Proceedings of the IEEE S\&P. $745-762$.

[79] Edward J. Schwartz, Thanassis Avgerinos, and David Brumley. 2011. Q: Exploit hardening made easy. In Proceedings of the USENIX SEC. 25-41.

[80] Hovav Shacham. 2007. The geometry of innocent flesh on the bone: Return-into-libc without function calls (on the x86). In Proceedings of the ACM CCS. 552-561.

[81] Hashim Sharif, Muhammad Abubakar, Ashish Gehani, and Fareed Zaffar. 2018. TRIMMER: Application specialization for code debloating. In Proceedings of the ACM/IEEE ASE. ACM, 329-339.

[82] Yan Shoshitaishvili, Ruoyu Wang, Christopher Salls, Nick Stephens, Mario Polino, Audrey Dutcher, John Grosen, Siji Feng, Christophe Hauser, Christopher Kruegel, and Giovanni Vigna. 2016. SoK: (State of) The art of war: Offensive techniques in binary analysis. In Proceedings of the IEEE S\&P. 138-157.

[83] Kevin Z. Snow, Fabian Monrose, Lucas Davi, Alexandra Dmitrienko, Christopher Liebchen, and Ahmad-Reza Sadeghi. 2013. Just-intime code reuse: On the effectiveness of fine-grained address space layout randomization. In Proceedings of the IEEE S\&P. 574-588.

[84] Raoul Strackx, Yves Younan, Pieter Philippaerts, Frank Piessens, Sven Lachmund, and Thomas Walter. 2009. Breaking the memory secrecy assumption. In Proceedings of the EuroSec.

[85] Chengnian Sun, Yuanbo Li, Qirun Zhang, Tianxiao Gu, and Zhendong Su. 2018. Perses: Syntax-guided program reduction. In Proceedings of the ICSE. 361-371.

[86] Debian The Universal Operating System. 2020. The unstable distribution ("sid"). Retrieved from https://www.debian.org/releases/sid/.

[87] László Szekeres, Mathias Payer, Tao Wei, and Dawn Song. 2013. SoK: Eternal war in memory. In Proceedings of the IEEE S\&P. 48-62.

[88] t0x0sh. 2014. A tool to help you write binary exploits. Retrieved from https://github.com/t00sh/rop-tool.

[89] The Chromium Projects. 2018. Testing and infrastructure. Retrieved from https://www.chromium.org/developers/testing.

[90] Caroline Tice, Tom Roeder, Peter Collingbourne, Stephen Checkoway, Úlfar Erlingsson, Luis Lozano, and Geoff Pike. 2014. Enforcing forward-edge control-flow integrity in GCC \& LLVM. In Proceedings of the USENIX SEC. 941-955.

[91] TIS Committee. 1995. Tool Interface Standard (TIS), Executable and Linking Format (ELF) Specification (v1.2). Retrieved from http://refspecs.linuxbase.org/elf/elf.pdf.

[92] Trail of Bits. 2019. McSema. GitHub. Retrieved from https://github.com/trailofbits/mcsema.

[93] Victor van der Veen, Dennis Andriesse, Enes Göktaş, Ben Gras, Lionel Sambuc, Asia Slowinska, Herbert Bos, and Cristiano Giuffrida. 2015. Practical context-sensitive CFI. In Proceedings of the ACM CCS. 927-940.

[94] Gregor Wagner, Andreas Gal, and Michael Franz. 2011. "Slimming" a Java virtual machine by way of cold code removal and optimistic partial program loading. Sci. Comput. Program. 76, 11 (Nov. 2011), 1037-1053.

[95] Ruoyu Wang, Yan Shoshitaishvili, Antonio Bianchi, Aravind Machiry, John Grosen, Paul Grosen, Christopher Kruegel, and Giovanni Vigna. 2017. Ramblr: Making reassembly great again. In Proceedings of the NDSS.

[96] Shuai Wang, Pei Wang, and Dinghao Wu. 2015. Reassembleable disassembling. In Proceedings of the USENIX SEC. 627-642.

[97] Zhe Wang, Chenggang Wu, Jianjun Li, Yuanming Lai, Xiangyu Zhang, Wei-Chung Hsu, and Yueqiang Cheng. 2017. ReRanz: A lightweight virtual machine to mitigate memory disclosure attacks. In Proceedings of the ACM VEE. 143-156.

[98] Richard Wartell, Yan Zhou, Kevin W. Hamlen, Murat Kantarcioglu, and Bhavani Thuraisingham. 2011. Differentiating code from data in x86 binaries. In Proceedings of the ECML-PKDD. 522-536.

[99] Debian Wiki. 2020. Using Symbols Files. Retrieved from https://wiki.debian.org/UsingSymbolsFiles.

[100] Ubuntu Wiki. 2019. Debug Symbol Packages. Retrieved from https://wiki.ubuntu.com/Debug\%20Symbol\%20Packages.

[101] David Williams-King, Graham Gobieski, Kent Williams-King, James P. Blake, Xinhao Yuan, Patrick Colp, Michelle Zheng, Vasileios P. Kemerlis, Junfeng Yang, and William Aiello. 2016. Shuffler: Fast and deployable continuous code re-randomization. In Proceedings of the USENIX OSDI. 367-382.

[102] David Williams-King, Hidenori Kobayashi, Kent Williams-King, Graham Patterson, Frank Spano, Yu Jian Wu, Junfeng Yang, and Vasileios P. Kemerlis. 2020. Egalito: Layout-agnostic binary recompilation. In Proceedings of the ASPLOS. 133-147.

[103] Windows Dev Center. 2018. PE Format. Retrieved from https://msdn.microsoft.com/library/windows/desktop/ms680547(v=vs.85) .aspx.

[104] Patrick Wollgast, Robert Gawlik, Behrad Garmany, Benjamin Kollenda, and Thorsten Holz. 2016. Automated multi-architectural discovery of CFI-resistant code gadgets. In Proceedings of the ESORICS. 602-620.

[105] Guoqing Xu. 2012. Finding reusable data structures. In Proceedings of the ACM OOPSLA. 1017-1034. 
[106] Andreas Zeller and Ralf Hildebrandt. 2002. Simplifying and isolating failure-inducing input. IEEE Trans. Softw. Eng. 28, 2 (Feb. 2002), $183-200$.

[107] Chao Zhang, Tao Wei, Zhaofeng Chen, Lei Duan, Laszlo Szekeres, Stephen McCamant, Dawn Song, and Wei Zou. 2013. Practical control flow integrity and randomization for binary executables. In Proceedings of the IEEE S\&P. 559-573.

[108] Mingwei Zhang and R. Sekar. 2013. Control flow integrity for COTS binaries. In Proceedings of the USENIX SEC. 337-352.

Received May 2020; revised October 2020; accepted December 2020

Digital Threats: Research and Practice, Vol. 1, No. 4, Article 19. Publication date: December 2020. 